\title{
Elongation of Flare Ribbons
}

\author{
Jiong Qiu ${ }^{1}$, Dana W. Longcope ${ }^{1}$, Paul A. Cassak ${ }^{2}$, and Eric R. Priest ${ }^{3}$ \\ ${ }^{1}$ Department of Physics, Montana State University, Bozeman MT, USA \\ 2 Department of Physics and Astronomy, West Virginia University, Morgantown WV, USA \\ ${ }^{3}$ School of Mathematics and Statistics, University of St. Andrews, Fife KY16 9SS, Scotland, UK \\ Received 2017 January 18; revised 2017 February 20; accepted 2017 February 25; published 2017 March 20
}

\begin{abstract}
We present an analysis of the apparent elongation motion of flare ribbons along the polarity inversion line (PIL), as well as the shear of flare loops in several two-ribbon flares. Flare ribbons and loops spread along the PIL at a speed ranging from a few to a hundred $\mathrm{km} \mathrm{s}^{-1}$. The shear measured from conjugate footpoints is consistent with the measurement from flare loops, and both show the decrease of shear toward a potential field as a flare evolves and ribbons and loops spread along the PIL. Flares exhibiting fast bidirectional elongation appear to have a strong shear, which may indicate a large magnetic guide field relative to the reconnection field in the coronal current sheet. We discuss how the analysis of ribbon motion could help infer properties in the corona where reconnection takes place.
\end{abstract}

Key words: magnetic reconnection - Sun: activity - Sun: flare - Sun: magnetic field

\section{Introduction}

Two-ribbon flares have been used as textbook examples demonstrating the standard flare reconnection configuration. The standard model is two dimensional (2D), which would imply simultaneous reconnection everywhere along the entire current sheet. However, this does not occur in nature, and all two-ribbon flares exhibit properties deviating from the 2D assumption. Even the most 2D-like two-ribbon flare arcade consists of discrete fine loops (Aschwanden \& Alexander 2001), each of them formed by a reconnection event. In this sense, reconnection is fragmented in space. The question is: do multiple reconnection events take place sporadically at several locations along the current sheet as a result of a global instability, or do adjacent reconnection events trigger one another in an organized manner? Observationally, many, though not all, flares exhibit localized reconnection events that are globally organized. This is characterized by the apparent elongation motion of flare ribbons in the lower atmosphere along the magnetic polarity inversion line (PIL) - the "zipper" motion-followed by an ordered spreading of flare loops anchored at the ribbons. These observations reflect a manifestation of energy release and the formation of flare loops by reconnection events in the corona successively along the PIL.

Spreading of flare ribbons or flare loops has been observed for several decades, and a vocabulary has been developed to describe this phenomenon. Vorpahl (1976) reported "sequential brightening" of flare soft X-ray emission along the axis of a flare arcade, at an apparent speed of $180-280 \mathrm{~km} \mathrm{~s}^{-1}$. Kawaguchi et al. (1982) and Kitahara \& Kurokawa (1990) reported "progressive brightenings" of short duration $\mathrm{H} \alpha$ emission along two flare ribbons, at a speed ranging from 100 to $500 \mathrm{~km} \mathrm{~s}^{-1}$. These authors interpreted the apparent along-the-ribbon motion as due to propagating magnetosonic waves, although the estimated wave speed is a few times greater than the observed ribbon-spreading speed.

Subsequent observers have reported a large number of flares with sequential brightenings of flare loops along the magnetic PIL. Motivated by the dawn-dusk asymmetry of magnetospheric substorms, Isobe et al. (2002) examined the "successive formation" of soft X-ray loops along the arcade axis observed by the Solar X-ray Telescope (Tsuneta et al.1991). They found 21 events with such characteristics, with a speed of $3-30 \mathrm{~km} \mathrm{~s}^{-1}$, substantially lower than those found in earlier case studies. They also found that 15 out of these 21 arcade events spread along the same direction as the reconnection electric field $\boldsymbol{E}$ in the corona, and the rest of them spread in the opposite direction. In a selective sample study, Tripathi et al. (2006) measured the "propagation" of flare EUV loops observed by the Extreme ultraviolet Imager Telescope at $195 \AA$ in 17 events, which were accompanied by erupting filaments. In these events, the measured propagation speed ranges from 13 to $111 \mathrm{~km} \mathrm{~s}^{-1}$, though mostly below $50 \mathrm{~km} \mathrm{~s}^{-1}$. They also found that the propagation speed of flare loops is larger in some events where filament motion is relatively fast. In their sample, 15 out of the 17 events exhibit unidirectional propagation as well as asymmetric filament eruption (e.g., eruption from one end), and the other two exhibit bidirectional propagation and symmetric filament eruption (e.g., eruption from the middle). Li \& Zhang (2009) also studied the "propagation" of flare loops observed by the Transition Region and Coronal Explorer (TRACE; Handy et al. 1999) at $171 \AA$ from 1998 to 2005, providing a much larger and unbiased sample that includes $151 \mathrm{M}$-class and $39 \mathrm{X}$-class flares. The measured speed ranges from 3 to $39 \mathrm{~km} \mathrm{~s}^{-1}$, in the same range as in Isobe et al. (2002). Li \& Zhang (2009) reported that about half of these events exhibit unidirectional propagation, and the other half exhibit bidirectional propagation.

Flare ribbons or kernels in the lower atmosphere outline the feet of flare loops or arcades, and are often brightened impulsively in optical and UV wavelengths before flare loops are visible in soft X-ray and EUV wavelengths. They are therefore instantaneous signatures of energy release by reconnection. The propagation of ribbon fronts or kernels along the PIL has been studied with much improved instrument tempospatial resolutions (Fletcher et al. 2004; Lee \& Gary 2008; Qiu 2009; Liu et al. 2010; Qiu et al. 2010; Cheng et al. 2012). Most of these are case studies, and the measured apparent motion speed, ranging between 15 and $70 \mathrm{~km}^{-1}$, is generally lower than those reported earlier by Kawaguchi et al. (1982) 
and Kitahara \& Kurokawa (1990), but higher than the measurements of loop propagation (Isobe et al. 2002; Li \& Zhang 2009).

Finally, apparent motion is also found in thick-target hard $\mathrm{X}$-ray footpoint sources observed by the Hard X-ray Telescope (HXT; Kosugi et al. 1991) and the Reuven Ramaty High Energy Solar Spectroscopic Imager (RHESSI; Lin et al. 2002). Bogachev et al. (2005) examined flares observed with $H X T$ at the M2 band (photon energy 33-53 keV) from 1991 through 2001, finding that out of 31 events that exhibit hard X-ray source motion, $11 / 8$ events show conjugate sources moving in the same/opposite direction along the magnetic PIL, the so-called "parallel or anti-parallel" motion. Using RHESSI observations, Krucker et al. (2003) observed one hard X-ray footpoint moving along the flare ribbon at a speed of $50 \mathrm{~km} \mathrm{~s}^{-1}$. Grigis \& Benz (2005) found the apparent motion of a pair of conjugate footpoint sources with a mean speed of $50-150 \mathrm{~km} \mathrm{~s}^{-1}$, with both sources moving in the same direction. In a more comprehensive sample study, Yang et al. (2009) examined 27 RHESSI M-X class flares that exhibit motion of hard X-ray footpoints, finding that the "parallel/antiparallel motion" is present in most of these events during the impulsive phase of the flare (defined by the rise of the GOES 1-8 А soft X-ray emission), which is sometimes followed by the "perpendicular motion" of the sources, taking place more frequently in the gradual phase (the decay of the GOES soft X-ray emission). A two-step footpoint motion pattern was also noted by Qiu (2009), Qiu et al. (2010), and Cheng et al. (2012). Specifically, Qiu et al. (2010) and Cheng et al. (2012) studied two X-class flares, and showed that the newly brightened flare ribbon fronts observed in UV $1600 \AA$ by TRACE and the hard X-ray footpoints observed by $H X T$ or RHESSI exhibit mostly consistent motion patterns in both wavelengths, first along the ribbon at a speed between $20-90 \mathrm{~km} \mathrm{~s}^{-1}$, and then perpendicular to the ribbon. However, Inglis \& Gilbert (2013) found an X-class flare showing a more complicated motion of the HXR footpoints, such as a reversal of motion along the UV ribbon (note that such a reversal of motion was reported in Cheng et al. 2012 as well), and a mismatch between the brightest UV emissions and the HXR source in the early stage of the flare. It is likely that UV emission is produced when the lower atmosphere is heated by either particle precipitation or thermal conduction (Coyner \& Alexander 2009), both resulting from reconnection energy release; on the other hand, hard X-ray sources tend to be mapped to locations where the energy flux is strong (Liu et al. 2007; Temmer et al. 2007). In this sense, the morphology of flare UV emissions may reflect a more complete mapping of reconnection energy release in the lower atmosphere.

Without directly measuring the motion speed or directionality, some other studies report a "shear motion" of conjugate flare footpoints. Here "shear" is defined as the angle made by the line connecting the conjugate footpoints with the line perpendicular to the magnetic PIL of the photospheric magnetogram. Su et al. (2007) found that $87 \%$ of the 50 selected M-X class two-ribbon flares observed by TRACE exhibit the trend that the footpoint shear is strong at the onset of the flare, and decreases as the flare evolves (see also Yang et al. 2009). Note that this shear measurement could arise from different motion patterns. It could be caused by conjugate footpoints moving in opposite directions along the ribbons, either approaching each other (shear decrease) or receding from each other (shear increase). This is different from the reported "zipper" effect, which refers to the loops (and the conjugate feet of the loops) propagating in the same direction. The shear change can be also produced by conjugate footpoints moving in the same direction (i.e., zipper motion) but at different speeds along the PIL.

All these observations of flare loop or ribbon motion along the ribbon direction indicate the three-dimensional (3D) nature of magnetic reconnection in solar flares. In a 2D framework, the along-the-ribbon motion is not present, and reconnection in the corona is characterized by a macroscopic electric field along the current sheet, assumed to be in the direction of the $\mathrm{PIL}, \boldsymbol{E}=-\boldsymbol{v}_{\text {in }} \times \boldsymbol{B}_{\text {in }} \approx \boldsymbol{v}_{\perp} \times \boldsymbol{B}_{n}$ (Forbes \& Lin 2000). Here $\boldsymbol{v}_{\text {in }}$ and $\boldsymbol{B}_{\text {in }}$ are the inflow velocity and magnetic field vectors at the boundary of the reconnection diffusion region in the corona. The perpendicular motion of the ribbon, also referred to as the "separation motion," is given by $\boldsymbol{v}_{\perp}$, and $\boldsymbol{B}_{n}$ is the normal component of the magnetic field in the chromosphere where the ribbon is formed, pointing either inward or outward from the solar surface. This perpendicular motion has been reported in most flare studies cited above, and its speed has been measured in flares to range from a few to a few tens $\mathrm{km} \mathrm{s}^{-1}$. With some assumptions or approximations made about the coronal inflow magnetic field $B_{\text {in }}$ and plasma density $\rho$ around the diffusion region, this motion speed is translated to a generic reconnection rate in terms of the Alfvén Mach number: $M_{a}=v_{\text {in }} / v_{a} \sim$ 0.001-0.1 (Isobe et al. 2005).

The apparent motion in the third dimension breaks the translational symmetry of the 2D model. It is entirely plausible that the 3D magnetic topology dictates the allowed flare connectivity and therefore the geometry of flare ribbons and loops (Aulanier et al. 2006). The question is one of dynamics: what mechanism determines the speed of such spreading of flare brightening or, alternatively, what physical properties characterize magnetic reconnection? A few different scenarios have been proposed to discuss the nature of this motion. They can be grouped into two categories.

Linear MHD waves have been invoked to explain the propagation of perturbations. Vorpahl (1976) proposed that magneto-acoustic waves are responsible for the sequential brightening of X-ray coronal loops along the axis of the arcade. If the axis of the arcade lies in the $y$ direction, the velocity component in this direction is given by

$$
2 v_{y}^{2}=v_{s}^{2}+v_{a}^{2} \pm \sqrt{\left(v_{s}^{2}+v_{a}^{2}\right)^{2}-4 v_{s}^{2} v_{a}^{2} \cos ^{2} \phi},
$$

where $v_{s}$ and $v_{a}$ are the local sound speed and Alfvén speed, respectively, and $\phi$ is the angle between the axis of the arcade and the magnetic field vector. We call the $y$-component of the magnetic field the guide field $B_{g}$, so that $\cos \phi=\hat{B} \cdot \hat{y}=$ $B_{g} / B$, where $B$ is the total magnetic field strength. The fast mode along the $y$ direction spreads at a speed greater than the Alfvén speed $v_{a}$ calculated with the total strength of the magnetic field $B$. Vorpahl (1976) assumed that the magnetic guide field $B_{g}=0$, and estimated the speed of the fast magnetosonic waves $v_{y}=\sqrt{v_{a}^{2}+v_{s}^{2}}$ to be a few times the observed speed of arcade spreading at $180-280 \mathrm{~km} \mathrm{~s}^{-1}$.

With the presence of the guide field $B_{g} \neq 0$, an Alfvén wave can also be supported traveling along the $y$-axis (Katz et al. 2010), 
at the Alfvén speed calculated using the guide field

$$
v_{y}=v_{a, g}=\frac{B_{g}}{\sqrt{\mu_{0} \rho}}=700 \frac{B_{g, 1}}{\sqrt{n_{9}}} \mathrm{~km} \mathrm{~s}^{-1} \text {, }
$$

where $\rho$ is the plasma mass density, $B_{g, 1}$ is the guide field in units of $10^{1} \mathrm{G}$, and $n_{9}$ is the electron number density in units of $10^{9} \mathrm{~cm}^{-3}$. Since the Alfvén speed is determined by only a component of the coronal magnetic field, $v_{y}$ could be smaller than the speed of magnetosonic waves by a factor of a few.

In these MHD wave scenarios, $v_{y}$ as estimated above is of order $10^{3} \mathrm{~km} \mathrm{~s}^{-1}$, given the general coronal properties. It should also be noted that MHD waves spread bidirectionally and symmetrically along the axis of the arcade.

Another macroscopic scenario based on a 3D framework suggests that 3D reconnection along Quasi-Separatrix Layers (QSLs; Priest \& Démoulin 1995) results in the spreading of the ribbon (Aulanier et al. 2006). 3D MHD numerical simulations have been conducted to qualitatively demonstrate the observed motion of flare kernels or loops (Masson et al. 2009; Aulanier et al. 2012; Janvier et al. 2013; Dudík et al. 2014; Savcheva et al. 2015). Some recent numerical simulations show the evolving QSLs "resulting from the expansion of a torus-unstable flux rope" (Aulanier et al. 2012; Dudík et al. 2014), which might qualitatively explain the relationship between filament/CME eruption and the propagation of flare loops observed by Tripathi et al. (2006) and Liu et al. (2010). It is not clear what controls the speed of the apparent motion in the topology models.

A different mechanism for ribbon spreading has been proposed due to microscopic current dynamics, motivated by observations in magnetospheric substorms, for which the guide field is typically small. It was proposed that drifting of currentcarrying particles leads to the spreading of reconnection along the current sheet, in the direction of the species carrying the current (Huba \& Rudakov 2002, 2003; Shay et al. 2003; Karimabadi et al. 2004; Lapenta et al. 2006). The spreading speed is

$$
v_{y}=\frac{B_{\mathrm{rec}}}{\mu_{0} n e \delta},
$$

where $B_{\text {rec }}$ is the reconnecting component of the magnetic field, $n$ is the electron number density in the current sheet, $e$ is the charge, and $\delta$ is the thickness of current sheet. This can be further expressed as

$$
v_{y}=5.0 \frac{B_{\mathrm{rec}, 1}}{n_{9} \delta_{5}} \mathrm{~km} \mathrm{~s}^{-1},
$$

where $B_{\text {rec, } 1}$ is in units of $10^{1} \mathrm{G}, n_{9}$ in units of $10^{9} \mathrm{~cm}^{-3}$, and $\delta_{5}$ in units of $10^{5} \mathrm{~cm}$. The thickness $\delta$ is related to the microphysics allowing reconnection. While it is unlikely that classical Sweet-Parker reconnection occurs (due to collisonless effects and secondary islands), we employ the Sweet-Parker model to get an estimate for the spreading speed. In the Sweet-Parker model, $\delta \sim L S^{-1 / 2}$, where $L \simeq 10^{9} \mathrm{~cm}$ is the global scale of the current sheet. A typical Lundquist number, $S \sim 10^{12}$, gives $\delta_{5} \sim 0.01$. This yields an estimated $v_{y}$ of at least $500 \mathrm{~km} \mathrm{~s}^{-1}$, which can be comparable with the propagation speed of MHD waves. Nevertheless, in this mechanism, $v_{y}$ is unidirectional; if electrons are the current carriers, $v_{y}$ is antiparallel to the direction of the current $\boldsymbol{J}$ in the current sheet.
Very recently, Shepherd \& Cassak (2012) conducted a theoretical analysis as well as numerical simulations to examine the propagation of reconnection along a current sheet as a function of a varying guide field. They argued that the spreading is controlled by whichever of the two mechanisms is faster, so that reconnection spreads bidirectionally at the Alfvén speed in a current sheet with a strong guide field $B_{g}$, asymmetrically with a weak guide field, or unidirectionally by current-carrying particles with a zero guide field.

As a result of the experimental, numerical, and theoretical works, it therefore appears that observations of elongation motion of flare ribbons may be used to infer properties of the coronal current sheet where reconnection takes place, such as the magnetic guide field or even the thickness of the current sheet. These properties are not directly measurable, but they are critical to our understanding of reconnection dynamics and energetics in solar flares. To be able to make an association between observations and models, the configuration of the flare needs to be relatively simple so that we can define the translational direction. For such a purpose, we analyze tworibbon flares taking place along a roughly straight magnetic PIL in active regions dominated by a bipolar configuration. We measure the apparent motion of ribbon brightening along the PIL, the presumed translational direction, as well as the inclination of flare loops with respect to the PIL to estimate the magnetic guide field relative to the total field of the flare loop. These measurements are then compared to examine whether the speed and direction of elongation are related to the shear to allow us to infer the current sheet properties. In the following, the method of analysis will be described in Section 2 and applied to three two-ribbon flares in Section 3. To enlarge the sample, we also include, in Section 3.4, results of other events analyzed previously. In Section 3.5, properties of ribbon motion are compared with magnetic properties of flare regions in search for understanding about governing mechanisms of ribbon elongation. Conclusions and discussions are given in the last section.

\section{Observations and Analysis}

This study will present analysis of three two-ribbon flares to illustrate apparent motion patterns of flare ribbon brightening. These events are selected because they demonstrate ordered ribbon motion along a rather collimated stretch of ribbons, allowing a $2.5 \mathrm{D}$ approximation and identification of the direction of the magnetic PIL as the translational direction, along which the ribbon-spreading speed can be measured. In addition to a comprehensive analysis of these three events, we will also review a few more flares previously analyzed and published, and summarize their properties together with the three cases.

The general information about these flares is given in Table 1 (Events 1, 2, and 3, respectively). All three flares analyzed in this study occur near the disk center in nearly bipolar magnetic configurations. The first event, C-class flare SOL2011-09-13, takes place in a growing active region consisting of a sunspot and a plage. The active region does not have a filament. For many hours before the flare, a soft X-ray sigmoid consisting of an arcade of sheared loops is visible in XRT on board Hinode (D. E. McKenzie 2014, private communication) as well as in the $335 \AA$ passband of the Atmosphere Imager Assembly (AIA; Lemen et al. 2012). Disruption of these loops is observed at the onset of the flare, and a new arcade of loops formed afterwards. This flare was also 
Table 1

Properties of Flare Ribbon Elongation

\begin{tabular}{|c|c|c|c|c|c|c|c|c|c|c|}
\hline & Date, Magnitude ${ }^{\mathrm{a}, \mathrm{b}}$ & Position & Time $^{c}$ & Direction $^{\mathrm{d}}$ & $\left\langle v_{\|}\right\rangle^{\mathrm{e}}$ & $\left\langle v_{\perp}\right\rangle^{\mathrm{f}}$ & $\theta_{f}{ }^{\mathrm{g}}$ & $B_{g} / B_{o}$ & $\left\langle B_{\mathrm{ph}}\right\rangle^{\mathrm{h}},\left\langle B_{c}\right\rangle^{\mathrm{i}}$ & $-\left\langle\nabla_{\|}|| B_{\mathrm{ph}} \mid\right\rangle^{\mathrm{j}}$ \\
\hline 1 & 2011 Sep 13 C2 & AR11289 N22W14 & $22: 51$ & anti-parallel $(\mathrm{P})$ & $-36 \pm 2$ & 0 & $39 \pm 4$ & 0.7 & $232 \pm 130,41 \pm 8$ & $1.7 \pm 0.1$ \\
\hline \multirow[t]{2}{*}{2} & 2011 Dec 26 C 3.7 & AR11384 N13W14 & $11: 18$ & parallel $(\mathrm{P})$ & $+81 \pm 2$ & 2 & $47 \pm 3$ & 0.9 & $99 \pm 113,15 \pm 4$ & $0.4 \pm 0.2$ \\
\hline & & & & parallel $(\mathrm{N})$ & $+98 \pm 2$ & 2 & $\ldots$ & $\cdots$ & $-107 \pm 121,15 \pm 4$ & $0.1 \pm 0.4$ \\
\hline \multirow[t]{2}{*}{3} & 2005 May 13 M8.0 & AR10759 N12E05 & $16: 34$ & bidirectional $(\mathrm{P})$ & $+29 \pm 3,-143 \pm 4$ & 8 & $11 \pm 2$ & 5.1 & $880 \pm 650,153 \pm 48$ & $52 \pm 2,-140 \pm 2$ \\
\hline & & & & bidirectional $(\mathrm{N})$ & $+80 \pm 6,-97 \pm 5$ & 14 & $\ldots$ & $\ldots$ & $-389 \pm 297,153 \pm 48$ & $-32 \pm 2,10 \pm 1$ \\
\hline \multirow[t]{2}{*}{4} & 2000 Jul 14 X5.7 & AR9077 N17E01 & $10: 21$ & anti-parallel (N) & $-65 \pm 4$ & 8 & $46 \pm 14$ & 1.0 & $-695 \pm 369,113 \pm 23$ & $-3.0 \pm 0.2$ \\
\hline & & & $10: 24$ & anti-parallel (P) & $-96 \pm 39$ & 8 & $50 \pm 10$ & 0.8 & $503 \pm 522,108 \pm 24$ & $-31 \pm 2$ \\
\hline \multirow[t]{3}{*}{5} & 2004 Nov 07 X2.0 & AR10696 N09W22 & $15: 44$ & bidirectional (P3) & $+13 \pm 2,-77 \pm 56$ & 10 & $<20$ & $>3$ & $779 \pm 307,271 \pm 53$ & $45 \pm 2$ \\
\hline & & & & bidirectional (P5) & $+77 \pm 4,-20 \pm 4$ & 10 & $\ldots$ & $\ldots$ & $360 \pm 175,271 \pm 53$ & $21 \pm 0.5$ \\
\hline & & & & bidirectional (N) & $+40 \pm 6,-92 \pm$ & 2 & $\ldots$ & $\cdots$ & $-369 \pm 231,271 \pm 53$ & $44 \pm 1,-4 \pm 0.5$ \\
\hline \multirow[t]{4}{*}{6} & 2005 Jan 15 X2.6 & AR10720 N13W04 & $22: 42$ & parallel $(\mathrm{P})$ & $+49 \pm 1\left(+55^{\mathrm{k}}\right)$ & 9 & $40 \pm 5$ & 1.2 & $1923 \pm 720,195 \pm 32$ & $96 \pm 10,-85 \pm 5$ \\
\hline & & & & parallel $(\mathrm{N})$ & $+35 \pm 1\left(+45^{\mathrm{k}}\right)$ & 22 & & & $-1093 \pm 392,195 \pm 32$ & $34 \pm 1.5$ \\
\hline & & & $22: 57$ & parallel $(\mathrm{P})$ & $+8 \pm 1$ & - & $75 \pm 2$ & 0.3 & $2227 \pm 385,338 \pm 29$ & $-139 \pm 5$ \\
\hline & & & & parallel $(\mathrm{N})$ & $+12 \pm 1$ & - & & & $-1425 \pm 584,338 \pm 29$ & $-105 \pm 3$ \\
\hline
\end{tabular}

Notes.

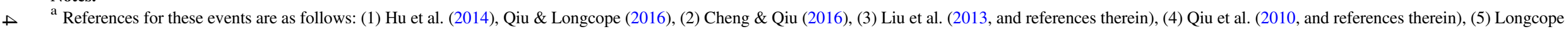
et al. (2007), Qiu (2009), (6) Liu et al. (2010), Cheng et al. (2012).

${ }^{\mathrm{b}}$ Magnitude is based on GOES classification.

${ }^{\mathrm{c}}$ Time refers to the start time when the elongation motion is measured.

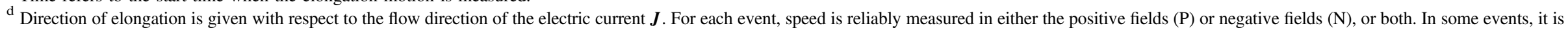
measured in multiple pieces of ribbons in the same polarity (such as P3 and P5 in event No. 5).

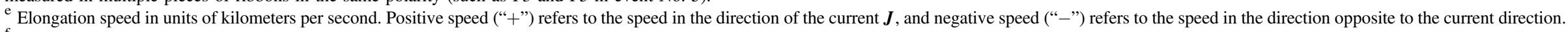

${ }^{\mathrm{f}}$ Mean perpendicular speed (in kilometers per second) of the entire ribbon measured when the ribbon is well-formed.

${ }^{\mathrm{g}}$ Inclination angle (degrees) measured from conjugate footpoints at the initial brightenings, except in event 1 , where the inclination angle measured from the first visible flare loops is used.

${ }^{\mathrm{h}}$ Mean photospheric longitudinal magnetic field (in Gauss) at the ribbon locations.

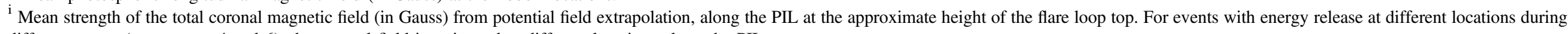
different stages (e.g., events 4 and 6), the coronal field is estimated at different locations along the PIL.

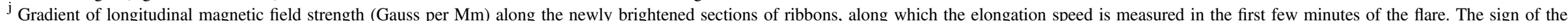

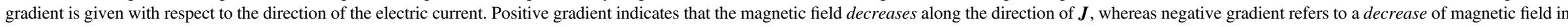
the direction opposite to $\boldsymbol{J}$.

${ }^{\mathrm{k}}$ These are speeds of HXR footpoints (Cheng et al. 2012). 

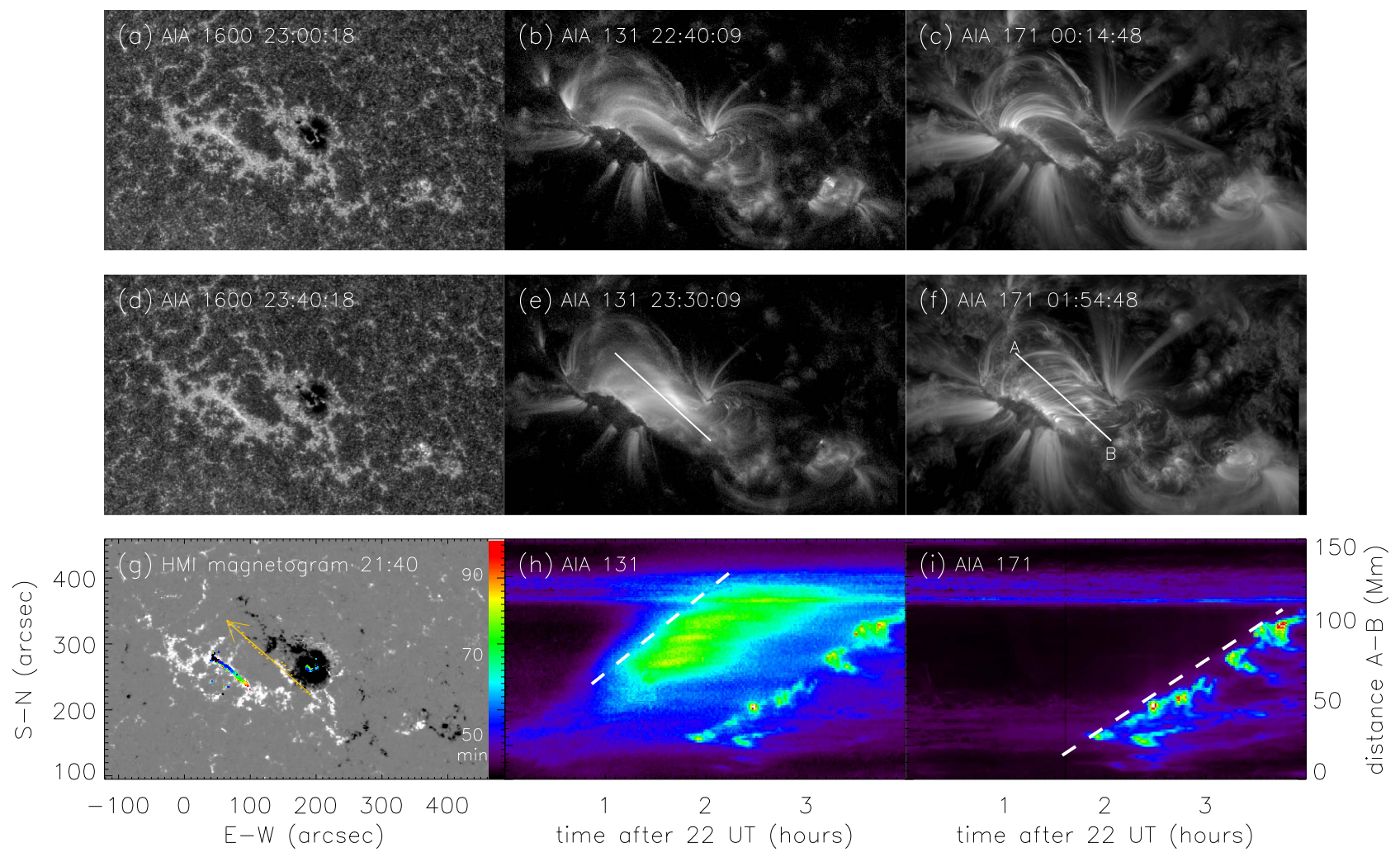

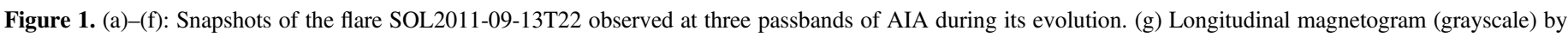

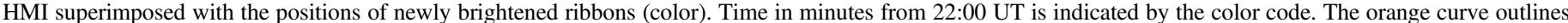

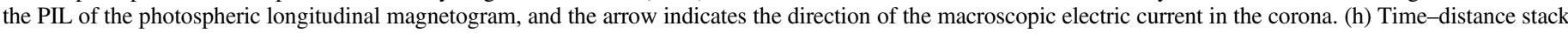

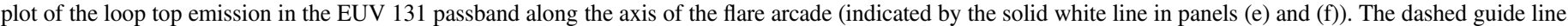

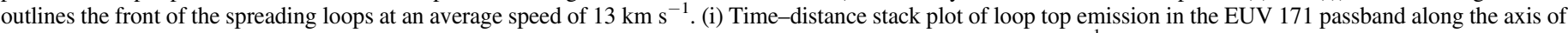
the flare arcade. The dashed guide line outlines the front of the spreading loops at an average speed of $10 \mathrm{~km} \mathrm{~s}$.

reported by $\mathrm{Hu}$ et al. (2014) and Qiu \& Longcope (2016). The second event, a C5.7 flare SOL2011-12-26, occurs in an active region without major sunspots. A remnant piece of a filament is visible in the active region; however, the filament is not observed to erupt, though a CME is observed by the Solar TErrestrial RElations Observatory(STEREO) and analyzed by Cheng \& Qiu (2016). The third event, an M8.0 flare SOL2005-05-13, occurs in an active region consisting of a sunspot and a plage. A filament sits along the magnetic PIL and partially erupted during the flare (Qiu \& Yurchyshyn 2005; Yurchyshyn et al. 2006; Liu et al. 2007, 2013; Kazachenko et al. 2009; Tripathi et al. 2009). The first two flares were observed by AIA, and the last by TRACE.

These flares exhibit two ribbons well-observed in the UV $1600 \AA$ passband on both sides of the PIL, with the shape of the ribbons outlining the PIL. Seen in Figures 1, 4, and 7, in each flare the ribbon brightening starts locally and then spreads along the PIL to form the full length of the ribbon-we call this motion the elongation motion. In some of these events, because of the asymmetry of magnetic fields of opposite polarities, the elongation motion is not symmetric in the two ribbons, with the brightening of one ribbon in the weaker magnetic field moving faster than the other in the stronger field so as to balance the positive and negative reconnection fluxes. The more well-known apparent motion of the flare ribbons, the perpendicular expansion away from the PIL, often also referred to as the separation motion, usually dominates after the elongation. In the framework of the 2D standard model, this separation motion has been related to the inflow speed at the boundary of the diffusion region in the corona, and therefore has been used to measure the mean reconnection rates in terms of the macroscopic electric field in the reconnection current sheet (Poletto \& Kopp 1986; Isobe et al. 2002; Qiu et al. 2002), although it remains unclear what exactly determines the electric field, or the rate of fast reconnection. The physical meaning of the first motion, the elongation, is much less understood, and this motion is the focus of this paper.

As in Qiu (2009), Qiu et al. (2010), and Cheng et al. (2012), we decompose the apparent motion of the front of the ribbon brightening into components along and perpendicular to the PIL. For each active region, the shape of the PIL relevant to the flare is outlined semi-automatically based on the pre-flare lineof-sight magnetogram and is then fitted by a polynomial, as shown by the orange symbols and curve, respectively, in Figures 1, 4, and 7. At a given moment of the flare, the trajectory of the outermost pixels of the ribbon is projected onto the PIL to define the positions of the two ends of the newly brightened ribbon front along the PIL, denoted by $l_{w}$ and $l_{e}$ for the two ends in the west and east, respectively. The length $l_{\|}=\left|l_{w}-l_{e}\right|$ along the PIL between the two ends is also measured. The area encompassed by the newly brightened ribbon pixels and the PIL is then divided by this length $l_{\|}$to yield the measurement of the mean perpendicular distance of the ribbon to the PIL, denoted by $\left\langle d_{\perp}\right\rangle$. The measurements were made using varying thresholds of UV counts (how many times the median or quiescent UV counts) to define ribbon fronts, and the standard deviation of these measurements is given as the uncertainty (error bars in the relevant plots). With these 

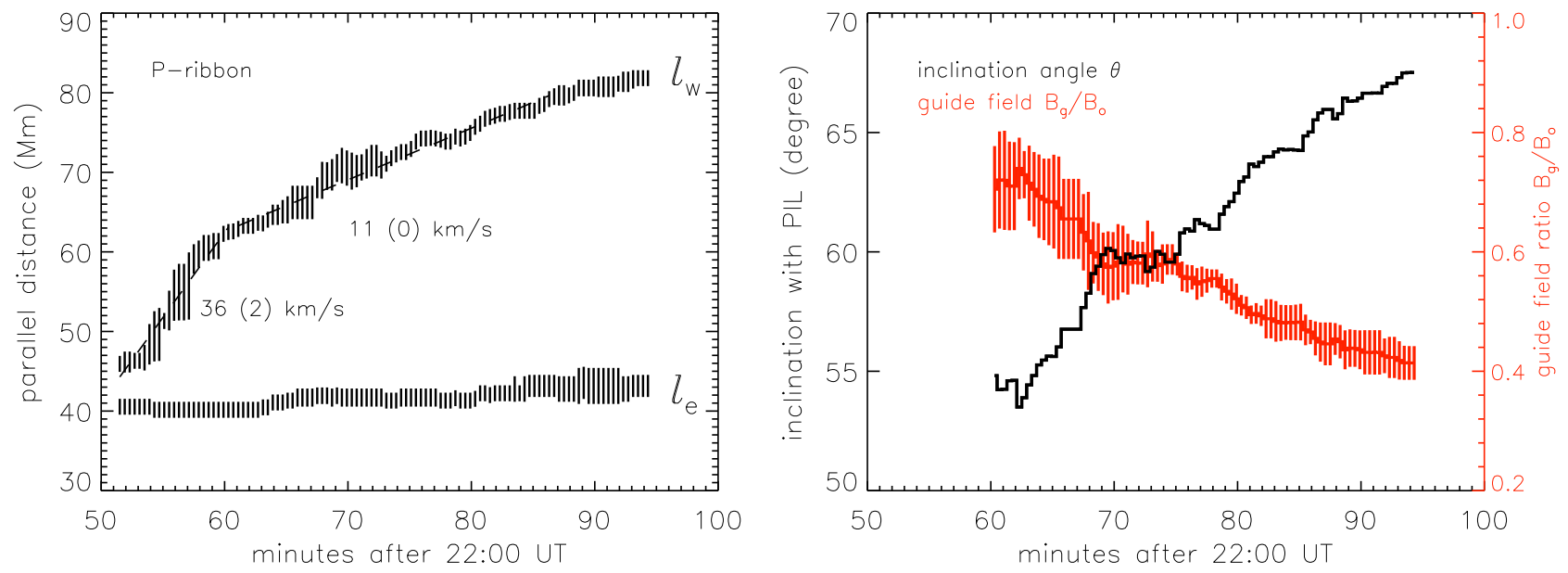

Figure 2. Left: position of the newly brightened front of the UV ribbon in the positive magnetic field along the PIL. Right: the inclination angle $\theta$ of the line connecting conjugate flare footpoints with respect to the PIL (black), and the presumed ratio of the guide field to the outflow field $B_{g} / B_{o}=\cot \theta$.

measurements, the apparent ribbon motion speed is found, $v_{w}=\Delta l_{w} / \Delta t, \quad v_{e}=\Delta l_{e} / \Delta t$, and $\left\langle v_{\perp}\right\rangle=\Delta\left\langle d_{\perp}\right\rangle / \Delta t$. In a strictly $2 \mathrm{D}$ version of the standard model, $v_{w}=v_{e}=0$ and reconnection in the corona is characterized by a macroscopic electric field along the current sheet, assumed to be in the direction of the PIL, $\boldsymbol{E}=-\boldsymbol{v}_{\text {in }} \times B_{\text {in }} \approx \boldsymbol{v}_{\perp} \times \boldsymbol{B}_{n}$. In practice, it has been assumed that $\boldsymbol{B}_{n}$ does not change significantly from the photosphere to the chromosphere, nor during the flare, so that $\boldsymbol{B}_{n}$ is usually taken from conventional photospheric magnetograms such as those provided by MDI and HMI. For active regions near disk center, it is also often approximated that $\boldsymbol{B}_{n}$ is the same as the longitudinal magnetic field.

To examine the possible relation of the ribbon motion to properties of the coronal current sheet, we also note the direction of the elongation motion with respect to the direction of the macroscopic electric current in the coronal current sheet. When a potential field anchored at photospheric bipoles is stretched upward, an electric current may be produced and its direction is determined by Ampere's law, $\boldsymbol{J} \propto \boldsymbol{\nabla} \times \boldsymbol{B}$. Whereas the exact magnetic field configuration or field strength is not known in the corona, the directionality of this field is reasonably deduced from photospheric magnetograms. In this way, the direction of the current density $\boldsymbol{J}$ is marked in the figures for the studied active regions, and the ribbon elongation motion is referred to as "parallel," "anti-parallel," or "bidirectional" with respect to this direction. ${ }^{4}$

We also report the inclination angle of post-reconnection loops with respect to the PIL. In a $2.5 \mathrm{D}$ approximation, the tangent of this angle would indicate the ratio of the magnetic outflow field $B_{o}$ and the magnetic guide field $B_{g}$, the component along the current which does not participate in reconnection. This inclination angle is measured in two ways. The first way, used in many previous studies, measures the angle between the line connecting the two ribbon fronts and the PIL determined from the photospheric magnetogram. When flare loops are observed, we also outline these loops and compute the angle between the loop and the PIL (determined from a potential field extrapolation) where they cross. To compare flare loops with a potential field, we extrapolate a

\footnotetext{
4 Some previous studies refer "parallel" or "anti-parallel" motion as conjugate footpoints moving in the same or opposite directions, different from the definition used in this study.
}

potential field and trace field lines from the same photospheric points as the potential-field loops, and find the angle where the projected field lines cross the PIL.

Finally, it should be noted that the emphasis of this study is to illustrate the global tempo-spatial pattern of ribbon brightening. A flare ribbon usually consists of a series of bright kernels but is not a continuous smooth patch of emission, as is also the case for the events in this study. According to current belief, these kernels are the feet of loops newly formed by reconnection. Energy released by an individual reconnection event propagates downward along the loop and heats the chromosphere at the footpoint kernels. It is also assumed that the footpoints of a loop do not move (i.e., line-tied assumption) over the flare timescale, hence the apparent motion of flare ribbon brightening refers to successive energy release and atmospheric heating at adjacent locations, and these brightenings map successive reconnection events in the corona down to the chromosphere.

\section{Apparent Motion of Flare Ribbons}

\subsection{Persistent Anti-parallel Elongation (2011 September 13)}

The C-class flare SOL2011-09-13T22:00 exhibits persistent elongation motion of the positive ribbon front, with little perpendicular expansion following the elongation. The left panels in Figure 1 show the flare morphology in the UV $1600 \AA$ band observed by AIA and the mapping of flare ribbons on a line-of-sight magnetogram obtained by HMI. Elongation of the ribbon in the plage of the positive magnetic field proceeds for nearly an hour. The distance of the ribbon brightening front along the PIL in both directions is measured and shown in Figure 2. The brightening spreads only in one direction (i.e., only $l_{w}$ grows) at a mean speed of $36 \mathrm{~km} \mathrm{~s}^{-1}$ in the first 5 minutes, and then at $11 \mathrm{~km} \mathrm{~s}^{-1}$ for the following hour. We note that the extension and motion of the ribbon in the sunspot of negative magnetic field is insignificant because of asymmetric magnetic field configuration at the two polarities, so the motion is not measured in the negative ribbon.

The ribbon brightening is then followed by sequentially formed flare loops first showing up in the northeast, and then spreading down along the PIL. Figures 1(b) and (e) show the arcade evolution in the AIA 131 A passband characterized by plasma emissions at $10 \mathrm{MK}$, and Figures 1(c) and (f) show the 
evolution of the flare loop arcade in the $171 \AA$ passband of plasma cooled to $1 \mathrm{MK}$ in about 90 minutes (Qiu \& Longcope 2016). The speed of the apparent spread of the loops can be estimated from the stack plots along the axis of the arcade. The slit $\mathrm{AB}$ crossing the loop tops is shown in panels (e) and (f), and the stack plots along $A B$ for the two passbands of the hot $131 \AA$ emission and cool $171 \AA$ emission are shown in panels (h) and (i), respectively. The two dashed guide lines suggest loop spreading speeds of 14 and $10 \mathrm{~km} \mathrm{~s}^{-1}$, respectively, following emission fronts at the two passbands. The measurements have also been made with other passbands and along slits crossing different parts of the loop arcade from the legs to the top, by either tracking the front or the maximum brightness of the time-distance stack plot. In four passbands, $131 \AA(10 \mathrm{MK}), 94 \AA$ (6 MK), $335 \AA$ ( $3 \mathrm{MK})$, and $171 \AA$ (1 MK), measurements tracking the front yield mean speeds of $13.9 \pm 0.7, \quad 11.2 \pm 1.6, \quad 9.8 \pm 1.8$, and $9.6 \pm 0.5 \mathrm{~km} \mathrm{~s}^{-1}$, respectively. Measurements tracking the peak brightness give mean speeds of $7.0 \pm 0.5, \quad 6.2 \pm 0.9, \quad 6.8 \pm 2.9, \quad$ and $9.6 \pm 0.3 \mathrm{~km} \mathrm{~s}^{-1}$, which are systematically smaller than the first measurements, except for the $171 \AA$ band. In short, the pattern of loop spreading is consistent with ribbon spreading, although the measured speed of the loops is slightly lower than ribbon spreading.

The bipolar magnetic field configuration in this event allows us to determine the direction of the electric current of the stretched bipolar field at the possible location of reconnection above the PIL, which is indicated by the arrow in Figure 1(g). In this event, the ribbon/loop spreading is anti-parallel with respect to the current $\boldsymbol{J}$. Interestingly, the event exhibits little perpendicular expansion, and we therefore do not measure $v_{\perp}$ here.

With the $2.5 \mathrm{D}$ approximation as illustrated by the arcade configuration, we may estimate the inclination angle $\theta$ of flare loops with respect to the PIL. The complementary angle of $\theta$ has been referred to as the shear angle in previous studies. Therefore, a smaller $\theta$ in this study refers to a stronger shear as defined in previous studies. The right panel of Figure 2 shows the evolution of the (footpoint) inclination $\theta_{f}$ defined as the angle between the line connecting conjugate footpoints (the brightening fronts in the two ribbons) and the PIL of the photospheric magnetogram. This angle gradually increases from $55^{\circ}$ to $70^{\circ}$ as ribbons and loops spread along the PIL, ${ }^{5}$ which is consistent with many previous reports of decreasing shear during the flare evolution.

Since both pre-flare and flare loops are observed in the EUV wavelengths, we also demonstrate the inclination angle of the observed loop top with the PIL of the coronal magnetic field, which is approximated by a potential field extrapolation using the photospheric magnetogram as the bottom boundary. This inclination angle is denoted by $\theta_{b}$ and $\theta_{a}$, referring to loops before and after the flare, respectively.

Pre-flare loops are visible in the $335 \AA$ A passband, suggesting that the typical temperature of the pre-flare arcade is about $3 \mathrm{MK}$. A set of loops is selected manually along the axis of the arcade observed in a $335 \AA$ image taken at 21:00 UT, about 1.5 hours before the flare onset. This is shown in Figure 3(a). The estimated inclination angle $\theta_{b}$ of these loops along the coronal PIL, illustrated by the black solid line, is plotted with the blue

\footnotetext{
5 The measurement of $\theta_{f}$ before 23:00 UT is not presented since it carries large uncertainties due to weak emission in the negative field of the sunspot.
}

dashed line in Figure 3(c). $\theta_{b}$ is estimated to vary between $50^{\circ}$ and $60^{\circ}$ along the PIL in the corona.

Flare loops form sequentially and are visible in multiple passbands (see Figure 1). They are well-observed in the $171 \AA$ passband with the best contrast. About 100 of these loops in the $171 \AA$ images, formed at different times at different locations along the PIL, are outlined manually, and a subset of them is illustrated in Figure 3(b). Color coding indicates the time when a loop is first visible in this bandpass, with violet and blue loops forming earlier than yellow and orange loops. Note that these loops start to appear more than an hour after the ribbon brightening due to elongated plasma heating and cooling in the corona (Qiu \& Longcope 2016). We estimate the angles made by the top of these flare loops with the PIL of the coronal magnetic field. Color symbols in Figure 3(c) show the inclination angle of these loops formed at different times along the PIL. It is shown that loops that formed earlier (cold colors) carry a larger shear (smaller $\theta_{a}$ ) down to $40^{\circ}$. Loops that formed later (warm colors) have decreased shear with $\theta_{a}$ up to $75^{\circ}$. The solid red curve shows the average inclination angle $\theta_{a}$ along the PIL, which grows westward as the flare ribbon and loops spread westward. Such an evolution trend and the range of inclination angles are consistent with the results from the footpoint measurements. The inclination angle of most flare loops is also larger than that of pre-flare loops.

In a $2.5 \mathrm{D}$ configuration, an inclination angle different from $90^{\circ}$ indicates the presence of a guide field $B_{g} \neq 0$, but does not necessarily indicate a non-potential magnetic loop. To compare the estimated inclination angle of pre-flare and flare loops with the potential field itself, we also calculate the angles of potential-field lines anchored at the flare ribbons at the points their projections cross the PIL. These angles $\left(\theta_{p}\right)$ are plotted in black symbols in Figure 3(c). The angle $\theta_{p}$ also grows during the westward propagation, but the potential-field loops generally make a larger angle than the flare loops. The comparison therefore suggests that, on average, flare loops are more sheared than the potential field and less sheared than the pre-flare arcade.

The flare loop inclination may be translated into the ratio of the guide field to the outflow field by the relation $B_{g} / B_{o}=\cot \theta$, assuming that $\theta$ is the same as $\phi$, the angle of the magnetic field line with the translational direction. In this event, at the start of the flare, the inclination angle is down to $40^{\circ}$, when the elongation speed is $36 \mathrm{~km} \mathrm{~s}^{-1}$. Afterwards, the average flare inclination varies from $50^{\circ}$ to $70^{\circ}$, and hence $B_{g} / B_{o} \approx 0.8-0.4$, as the flare evolves with a steady ribbon elongation at $11 \mathrm{~km} \mathrm{~s}^{-1}$ for about an hour.

In summary, we find this event is a special example of slow and persistent spreading of reconnection along the PIL with a relatively weak shear. Note that this flare does not exhibit significant expansion of the ribbon perpendicular to the PIL, suggesting that the reconnection site might not rise in the corona as typically assumed in the standard flare model. It is notable that a potential-field source-surface model with a source surface at $2.5 R_{\odot}$ reveals this $\mathrm{AR}$ to have several unusual properties. It lies squarely underneath a closed separatrix dome whose null point lies practically at the source surface: $r=2.3 R_{\odot}$. It is possible that an eruption opened this dome, which then reformed through reconnection that occurred unusually high in the corona, which proceeded unusually slowly, with little notable outward expansion. 

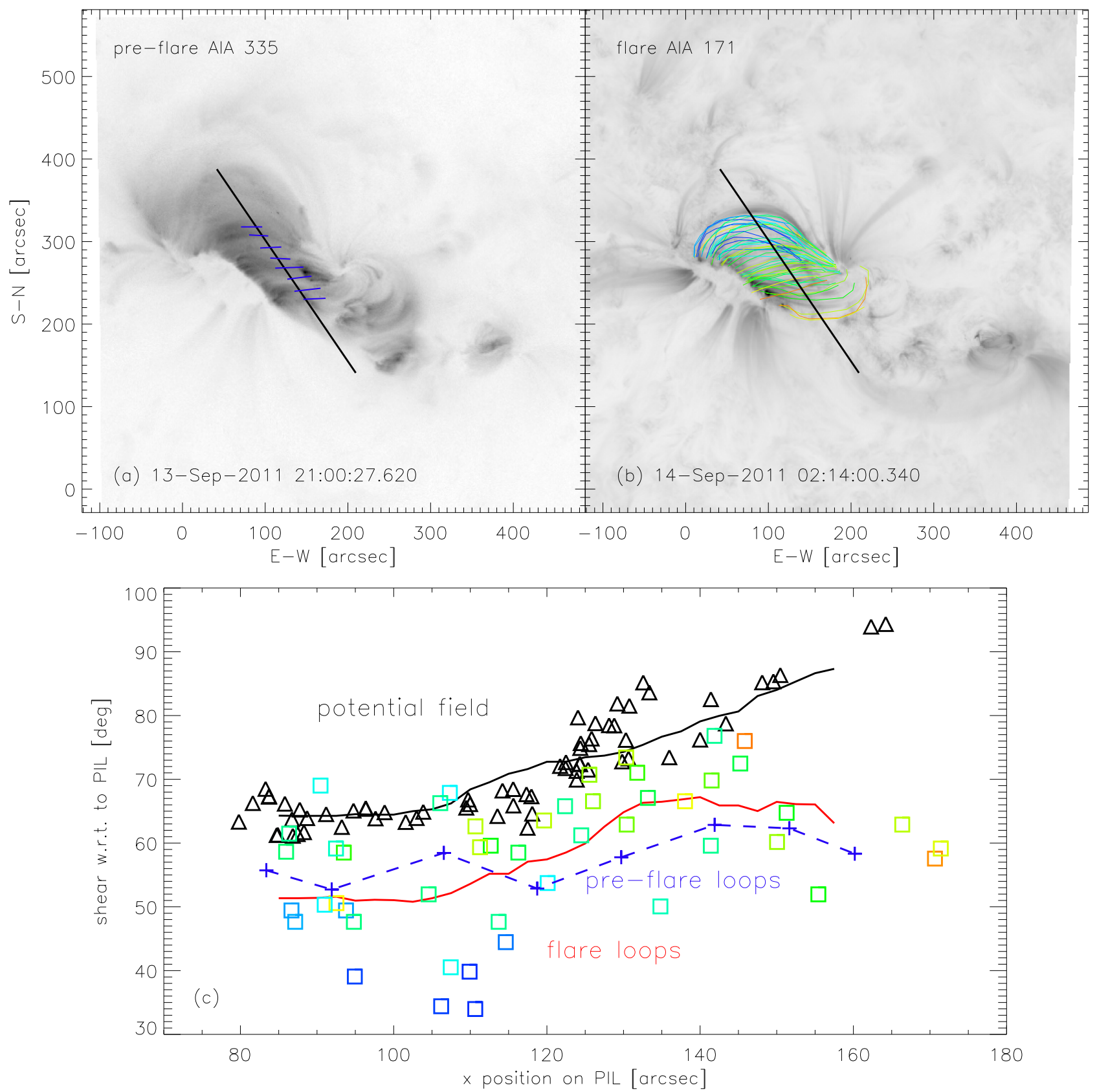

Figure 3. Top left: pre-flare loop arcade at 21:00 UT observed in the AIA $335 \AA$ Asssband, with the loop tops outlined in blue bars, and the black solid line indicating the magnetic PIL at the estimated height of the loop top. Top right: flare loop arcade at 02:14 UT observed in the AIA 171 A passband, with sequentially formed flare loops outlined in color curves (cold-color loops form earlier than warm-color loops), and solid line indicating the magnetic PIL at the estimated height of the loop top. Bottom: the inclination angle of the pre-flare $\left(\theta_{b}\right.$, blue dashed line) and flare loops $\left(\theta_{a}\right.$, color symbol) along the PIL. The red curve shows the average loop inclination along the PIL. Black symbols show the inclination angle $\theta_{p}$ of the potential-field lines anchored at the ribbon pixels, and the black curve shows the average potential field inclination along the PIL.

This example reveals several other properties not seen in previous studies. Most remarkably, the measured shear and its evolution from the footpoint brightenings with respect to the photospheric PIL is largely consistent with the measurement of flare loops with respect to the coronal PIL. It is also found that the motion pattern derived from the UV ribbon brightening is consistent with that derived from flare loops. Despite the asymmetry in the motion of conjugate footpoints, the flare arcade exhibits a rather homogeneous motion, suggesting that the coronal magnetic field is indeed more homogeneous than the photospheric distribution. On the other hand, the measured speed of flare loops is systematically lower than the speed of the footpoint motion, suggesting that the timescale of coronal loop spreading may be convolved with hydrodynamic timescales due to plasma heating and cooling in the corona.

\subsection{Fast Parallel Elongation (2011 December 26)}

In the next example, we observe the elongation of flare ribbons parallel to the direction inferred for the electric current along the PIL. The two-ribbon flare SOL2011-12-26T11:23 exhibits two nearly symmetric flare ribbons, both of which start to brighten from the southwest end of the AR and spread along the PIL toward the northeast. The left panels of Figure 4 show 

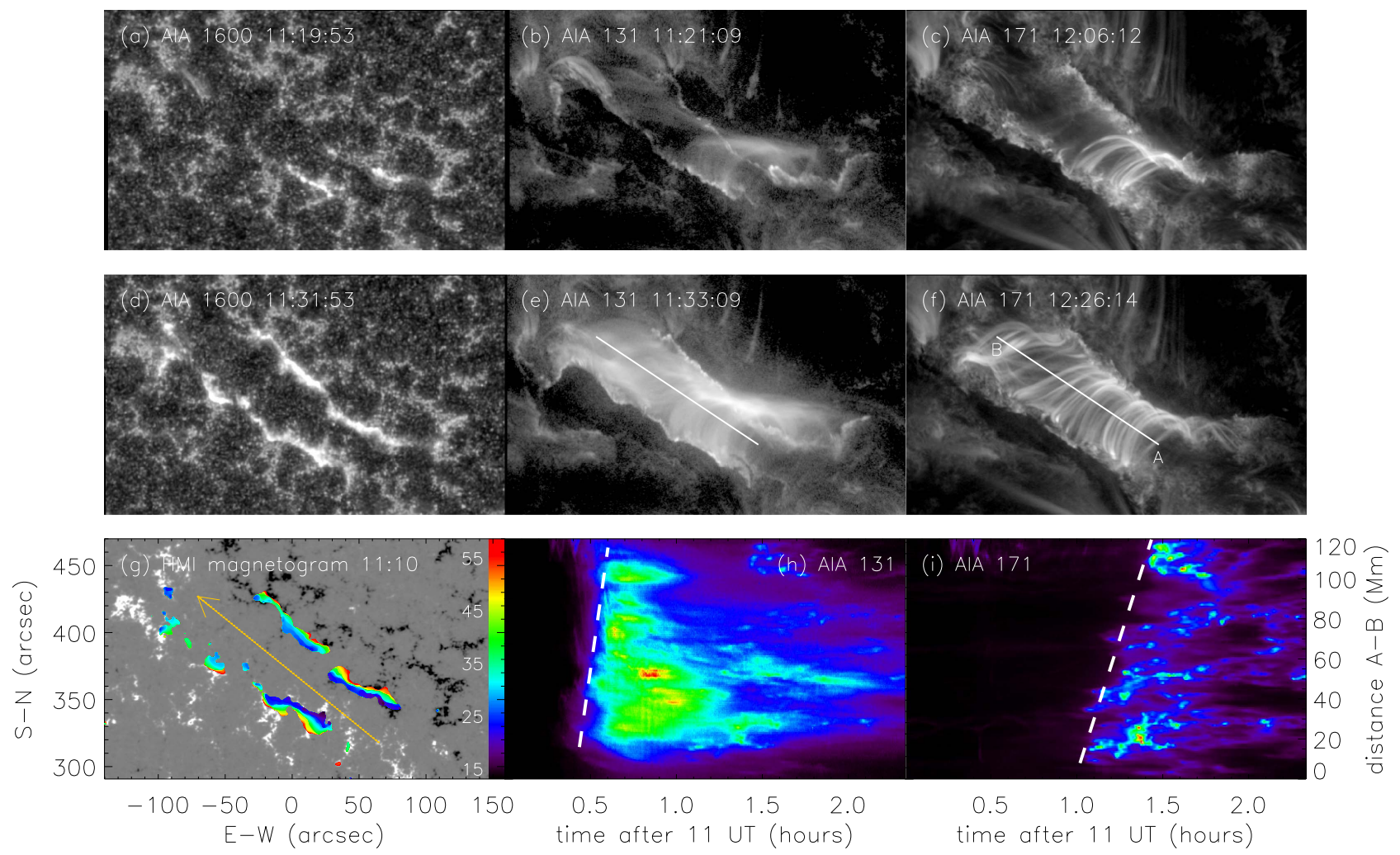

Figure 4. Same as Figure 1, for the flare SOL2011-12-26T11:23. The guide line in panel (h) suggests spreading of loops in the $131 \AA$ passband at the mean speed of $110 \mathrm{~km} \mathrm{~s}^{-1}$, and that in panel (i) indicates spreading of loops in the $171 \AA$ passband at the mean speed of $50 \mathrm{~km} \mathrm{~s}^{-1}$.

the evolution of the two ribbons; in both ribbons, the brightening spreads along the PIL in one direction (i.e., only $l_{e}$ grows) for about 10 minutes at the mean speed of 81 and $98 \mathrm{~km} \mathrm{~s}^{-1}$ for the positive and negative ribbons, respectively. These speeds are an order of magnitude greater than in the first event.

Again, for this flare, the flare arcade is observed in multiple wavelengths, allowing us to measure the speed of the spreading loops. The panels in the middle column show the evolution of the flare loops in the $131 \AA$ passband and the stack plot along a slit crossing the loop tops along the axis of the arcade; the right column shows observations and measurements in the 171 $\AA$ passband. For this event, the measured speed of the spreading hot (131 $⿱$ ) loop fronts is very similar to the speed of the ribbon spreading, of $110 \mathrm{~km} \mathrm{~s}^{-1}$; however, at the low temperature (1 MK) passband, the apparent motion of the cool loop front is a lot slower, at only $50 \mathrm{~km} \mathrm{~s}^{-1}$, suggesting that timescales of the hydrodynamic evolution (e.g., cooling) of loop plasmas have significantly affected the apparent speed of spread.

The active region hosting the two-ribbon flare is primarily bipolar with nearly symmetric positive and negative fields, both located in plages. The direction of the electric current is derived and indicated in the bottom left panel in Figure 4. The ribbon elongation is parallel to $\boldsymbol{J}$, which is different from the first event.

Also different from the first event, in this flare, the ribbon separation motion is observed, and the mean perpendicular speed in the positive ribbon is about $10 \mathrm{~km} \mathrm{~s}^{-1}$ in the first 10 minutes. Afterwards, as the two ribbons have fully developed and elongation motion has stopped, the separation motion continues at a mean speed of about $2 \mathrm{~km} \mathrm{~s}^{-1}$ in both ribbons for another 30 minutes.

In a similar manner, we estimate the inclination angle from newly brightened conjugate footpoints. Figure 5 shows that this angle $\theta_{f}$ changes from $45^{\circ}$ to nearly $90^{\circ}$ within 20 minutes, reflecting a more significant shear variation compared with the first event. From this angle, the estimated $B_{g} / B_{o}$ varies from 1 at the start of the flare to 0 .

Shear angles can also be estimated with flare loops. More than 200 flare loops are manually outlined in the $171 \AA$ images and shown in Figure 6(a). The right panel shows the measurement of inclination along the PIL of the coronal potential field, with cold colors indicating loops formed earlier than warm-color loops. In this event, because loops form quickly along the PIL in the initial phase and then "grow" upward in a nearly $2 \mathrm{D}$ manner, the measurement of inclination along the PIL does not seem to show a clear pattern due to a mixture of loops that formed earlier and later at the same PIL position, but there is clear indication that loops that formed earlier (cold-color) are, on average, more sheared, $\theta_{a} \approx 45^{\circ}-65^{\circ}$, than loops that formed later (warm color), with $\theta_{a} \approx 60^{\circ}-90^{\circ}$. Again, the range and evolution of the inclination of flare loops are remarkably consistent with the footpoint measurements.

The inclination angle of potential-field loops anchored at the ribbons is also estimated. In the west where ribbon brightening starts, flare loops are more sheared than potential loops; in the east, however, the small inclination angle of the potential field may be caused by the change in height of the loops, where the orientation of the PIL may have changed.

This flare is associated with a partial halo CME wellobserved by STEREO (Cheng \& Qiu 2016). The CME is first 

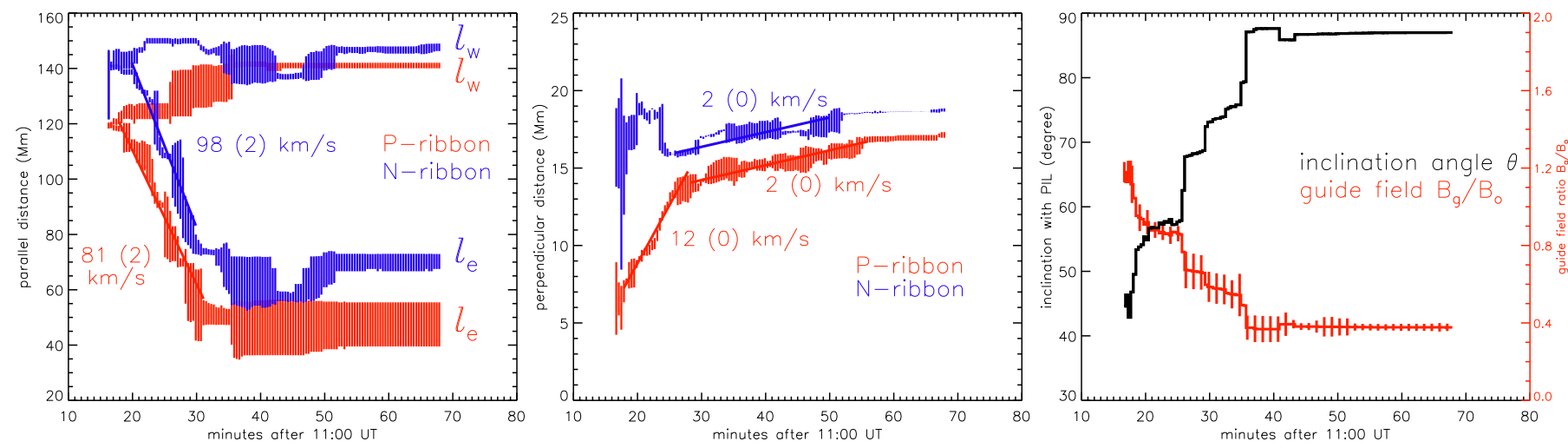

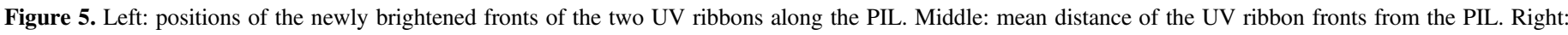

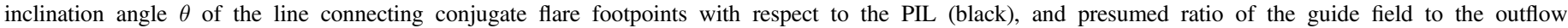
field $B_{g} / B_{o}=\cot \theta_{f}$.

detected at 11:06 UT, and appears to rise at 11:10 UT when brightening of UV ribbons starts. An analysis by Cheng \& Qiu (2016) shows that CME height evolves with reconnection measured in ribbons. The AIA disk observations show a faint remnant of a low-lying filament which did not erupt. The STEREO $304 \AA$ images also did not show filament eruption prior to the CME. It is observed that some activation of the filament remnant, including brightening and flows along the axis, is present at the northeast end of the filament, yet flare ribbon brightening starts at the southwestern end. Thus, it is not clear whether and how the global dynamics associated with the $\mathrm{CME}$ eruption would govern the ribbon elongation.

\subsection{Fast Bidirectional Elongation (2005 May 13)}

The third event is a well-studied M8.3 two-ribbon flare, SOL2005-05-13, which has been analyzed by Yurchyshyn et al. (2006), Qiu \& Yurchyshyn (2005), and Liu et al. (2007), as well as modeled by Kazachenko et al. (2009) and Liu et al. (2013). The flare was observed by TRACE mostly in the $1600 \AA$ A bandpass with a very high cadence of $3 \mathrm{~s}$ when the flare mode was switched on. Figure 7 shows the evolution of the two ribbons. Measurements of ribbon motion are plotted in Figure 8. For this flare, ribbon brightening starts at the middle in both ribbons, and spreads in both directions along the curved PIL. Spreading of the ribbon in the weak and negative magnetic field proceeds nearly symmetrically in both directions with an average speed of $v_{w} \approx v_{e} \approx 100 \mathrm{~km} \mathrm{~s}^{-1}$. In the strong positive magnetic fields inside a sunspot, the ribbon spreads asymmetrically; it moves more quickly toward the penumbra with $v_{e} \approx 80 \mathrm{~km} \mathrm{~s}^{-1}$, while in the opposite direction into the umbra, the ribbon spreading is much slower $v_{w} \approx 20 \mathrm{~km} \mathrm{~s}^{-1}$. We recognize that there are large uncertainties in the measurements of the speed because of the very low cadence (80-170 s) of TRACE observations at the start of the flare, when we only have 2-3 measurements as ribbon spans over a long distance. Because of the low cadence, it is also seen that when the ribbon starts to brighten, an extended section, rather than a compact kernel, of the ribbon brightened. Nevertheless, it can be observed that ribbon spreading is bidirectional from both ends of the section at a relatively high speed of order $100 \mathrm{~km} \mathrm{~s}^{-1}$.

In this event, perpendicular expansion of the ribbons is also observed, and $\left\langle v_{\perp}\right\rangle$ is measured and presented in the middle panel, showing that the perpendicular expansion dominates when elongation has stopped. The mean speed of this expansion is $10 \mathrm{~km} \mathrm{~s}^{-1}$ initially, more than an order of magnitude slower than the elongation motion. Later on when elongation has stopped, the ribbons still expand perpendicularly at a mean speed of $3 \mathrm{~km} \mathrm{~s}^{-1}$. The mean electric field due to such an expansion is derived to be $5 \mathrm{~V} \mathrm{~cm}^{-1}$ at about 16:40 UT, 10 minutes after the flare onset, and decreases afterwards as the perpendicular expansion slows down.

Finally, it would be interesting to learn how flare loops are sheared with respect to the PIL. Unfortunately, TRACE observations of flare loops in this event are scarce, so we only measure the inclination of the conjugate ribbon brightenings with respect to the photospheric PIL. We assume the connectivity between newly brightened ribbon fronts, and measure the change of the inclination angle with respect to the PIL. Because of bidirectional spreading of the ribbons, each ribbon has two newly brightened fronts. Ribbon observations alone do not provide the connectivity between two pairs of the fronts. The inclination is therefore measured by assuming both connectivities, and the measurements are presented in Figure 8(c). For yet another ambiguity, the PIL in this region is curved rather than nearly straight. In spite of these uncertainties, it is clear that this flare configuration possesses the largest shear among the three events. If we translate this inclination angle to the guide field relative to the outflow field, $B_{g} / B_{o} \approx 5$ initially, and then gradually decreases to unity.

A filament is clearly visible along the PIL (Yurchyshyn et al. 2006). It disappears during the flare, and shows up again hours after the flare. There is ambiguity as to whether the filament partially erupts (Tripathi et al. 2009) or does not disrupt at all but just experiences thermal disappearance (Liu et al. 2007). Again, it is not clear how filament activity would be related to the bidirectional spreading of ribbons.

Of the three flares, this event is the most energetic, and hard $\mathrm{X}$-ray emission up to $100 \mathrm{keV}$ is observed by RHESSI. Superimposed in Figure 8(c) is the RHESSI observed hard $\mathrm{X}$-ray data counts in $25-50 \mathrm{keV}$, which is most likely produced by non-thermal electron beams impacting the chromosphere (Liu et al. 2013). The peak of this non-thermal emission occurs at 16:43 UT. HXR spectral analysis (not shown here) also suggests that, at this moment, the photon spectrum is hardest. At this time, elongation of the ribbons has mostly stopped, or the ribbon has attained its maximum length, and the perpendicular expansion starts to slow down. The observation that HXR emission peaks after the elongation phase is consistent with an earlier report by Qiu et al. (2010) on the Bastille day event. From these few examples, it therefore 

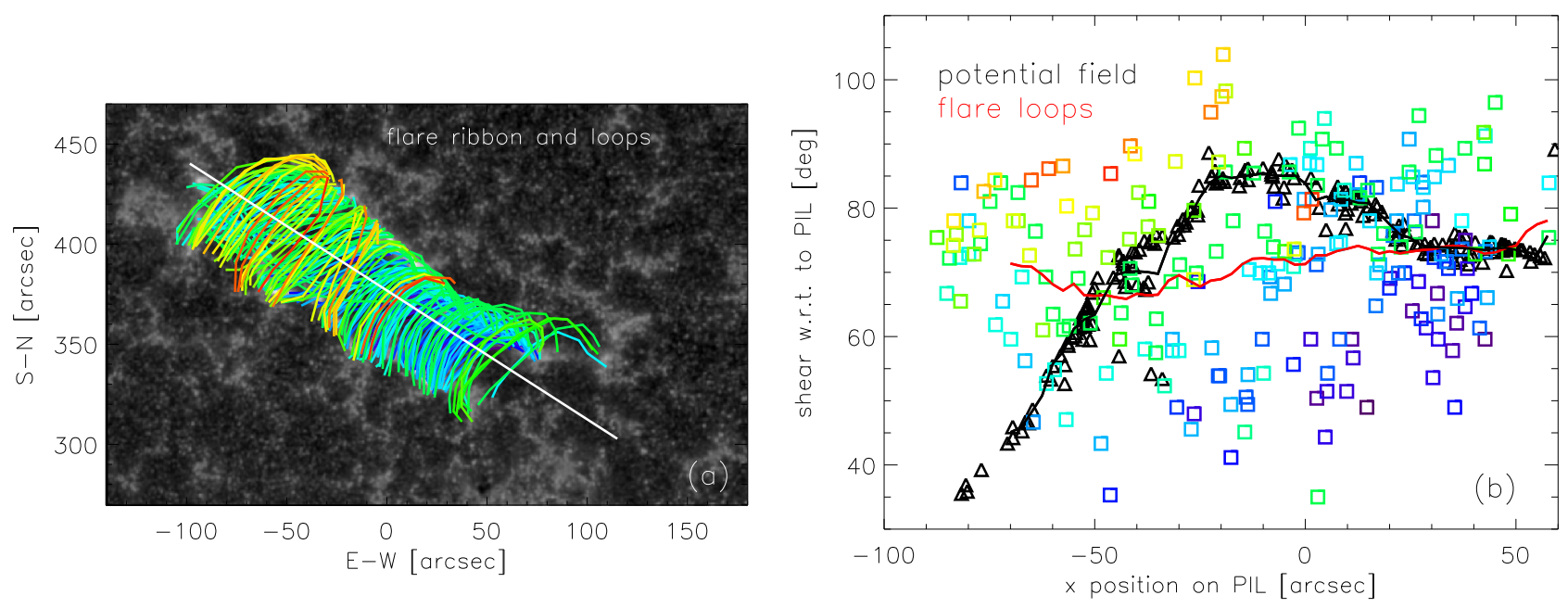

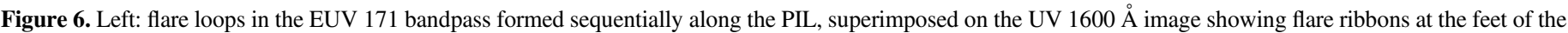

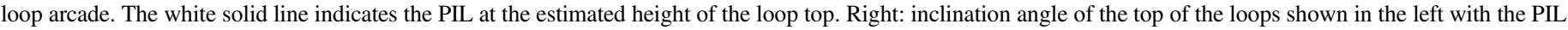
at the estimated height of the loop top, as in Figure 3(c).

appears that significant non-thermal particle production usually occurs after the elongation phase.

\subsection{Other Cases}

The events studied above provide examples of different kinds of ribbon elongation, one showing persistent slow antiparallel elongation and one with fast parallel elongation, both residing in magnetic fields of low to moderate shear, and the last event with fast bidirectional elongation in a strongly sheared configuration. In addition to these cases, we summarize the results of ribbon motion in other events presented in previous studies. These include the SOL2000-07-04 X5.7 flare (Bastille day flare) analyzed in Qiu et al. (2010, and references therein), the SOL2005-01-15 X1.5 flare analyzed by Liu et al. (2010) and Cheng et al. (2012), and the SOL2004-11-07 X2.0 flare analyzed by Longcope et al. (2007) and Qiu et al. (2009). Apparent ribbon motions have been measured in all of these flares. The first two flares each show two stages of energy release taking place at different locations along the PIL, and motion is measured separately in each stage. For the last flare, flare ribbon brightening starts at multiple locations and is tracked separately at these different places. All these events were observed in the UV $1600 \AA$ bandpass by TRACE. The observation cadence of the SOL2000-07-04 X5.7 flare is about $30 \mathrm{~s}$, tat of the SOL2004-11-07 X2.0 event and the SOL200501-15 X1.5 event is $7 \mathrm{~s}$ and $2 \mathrm{~s}$, respectively.

Figure 9 gives a general view of these three flares. For each flare, positions of the newly brightened ribbon fronts are plotted on a longitudinal magnetogram by MDI, with colors from violet to red indicating the time lapse. The definition of newly brightened ribbon fronts depends on the threshold data counts. In this figure, pixels with counts larger than 10 times the quiescent background are plotted. To measure the ribbon motion, a few thresholds are used, and the position of the newly brightened ribbon fronts is the mean measurement with these thresholds. Note that in this paper, we only plot evolution of the ribbon fronts in the first few minutes to focus on the elongation, which only occurs for a short time after the flare onset. The apparent motion of ribbon fronts, including the separation motion, over the entire flare duration, has been presented in previous studies.
The SOL2000-07-04 X5.7 flare (event 4 in Table 1) starts from the western end of the active region shortly after 10:00 UT, which is initiated by a filament eruption off that location. The observing cadence of $\geqslant 30 \mathrm{~s}$ does not allow us to track the motion of brightening reliably at the start of the flare. From 10:20 UT, a second stage of energy release takes place, and the flare spreads eastward toward the center of the active region, as indicated by the color map in Figure 9(a). It is noted that brightening starts at a couple of locations along each ribbon, and at some locations of strong emission, an organized pattern of elongation is observed. In particular, between 10:20:44 and 10:22:59 (violet to blue), eastward spreading is seen in both ribbons in the west of the AR. The mean speed of spreading is $65 \mathrm{~km} \mathrm{~s}^{-1}$, which is more reliably measured in the negative ribbon, and the uncertainty is simply the standard deviation of the linear fit to derive the mean speed. Afterwards, eastward elongation is observed further toward the east of the AR, especially in the positive ribbon. From 10:24:10 to 10:26:56 (green to red), the mean speed of the spreading in the positive ribbon is $96 \mathrm{~km} \mathrm{~s}^{-1}$. We note that a large section of the negative ribbon during this stage is brightened simultaneously with a less clear pattern of motion, so we do not measure the speed there. Fletcher \& Hudson (2001) have observed the same pattern of ribbon evolution. From the magnetogram, the macroscopic electric current along and above the PIL runs westward. ${ }^{6}$ Therefore, the overall trend of ribbon elongation is anti-parallel in this event. The inclination angle of the postreconnection connectivity is estimated to be below $50^{\circ}$ at around 10:21 UT, and $50^{\circ} \pm 10^{\circ}$ from 10:25 to 10:27 UT (Qiu et al. 2010), yielding $B_{g} / B_{o} \approx 1.0-0.8$.

The SOL2004-11-07 X2.0 flare (event 5) starts at the PIL, with brightening occurring at a few places. For this flare, Qiu (2009) measured the motion of brightening in a few magnetic cells throughout the flare duration. Figure 9(b) displays the ribbon brightening only in the first 2.5 minutes. It shows that in the positive polarity, brightening starts at three places and spreads along the PIL from two locations. In the westmost patch (P5 in Qiu 2009), elongation is bidirectional at $77 \mathrm{~km} \mathrm{~s}^{-1}$ westward and $20 \mathrm{~km} \mathrm{~s}^{-1}$ eastward. The middle patch (P3 in Qiu 2009)

\footnotetext{
6 Note that Qiu et al. (2010) mistakenly gave the direction of the reconnection electric field as eastward.
} 

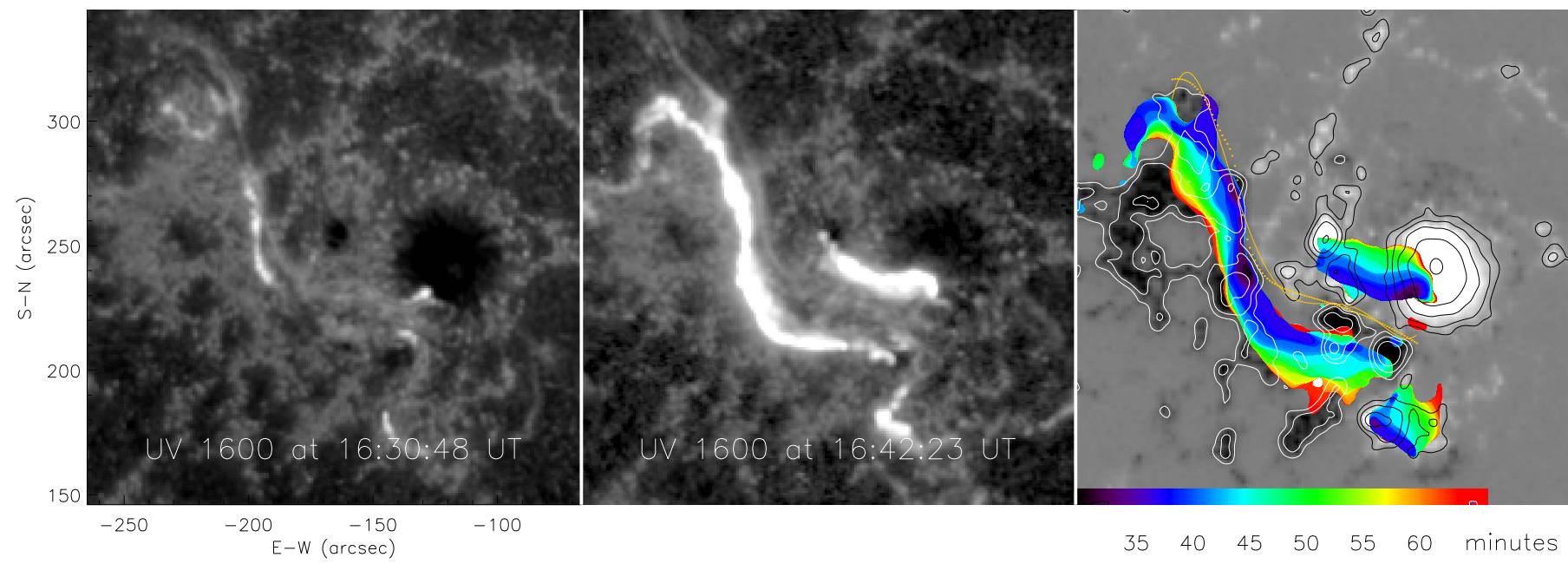

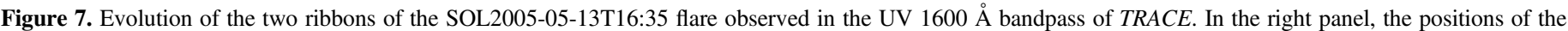
newly brightened ribbon fronts are superimposed on a longitudinal magnetogram by MDI, with the time lapse given by the color code.

spreads mostly eastward at a mean speed of $77 \mathrm{~km} \mathrm{~s}^{-1}$. In the negative polarity, brightening starts in one patch, which spreads bidirectionally at a speed of $40 \mathrm{~km} \mathrm{~s}^{-1}$ westward and $92 \mathrm{~km} \mathrm{~s}^{-1}$ eastward. The motion pattern in this event is therefore rather complex, with different behaviors at different locations. The macroscopic electric current runs eastward in this flare. There is a large uncertainty in the measurement of the initial inclination because the negative ribbon may be connected to different patches in the positive polarity. Nevertheless, this initial inclination angle can be estimated to be smaller than $20^{\circ}$, yielding $B_{g} / B_{o}$ greater than 3 .

The SOL2005-01-15 X1.5 flare (event 6) also exhibits two stages of energy release at two different locations along the PIL (Cheng et al. 2012). The ribbon brightening fronts are plotted in Figures 9(c) and (d), respectively, for these two stages. From 22:41:52-22:45:07, brightening occurs in the west of the AR with organized eastward elongation at average speeds of 49 and $35 \mathrm{~km} \mathrm{~s}^{-1}$ in the positive and negative ribbons, respectively. This flare was also observed by RHESSI. Cheng et al. (2012) found two HXR kernels at energies above $25 \mathrm{keV}$, which are located at the maximum UV emission along the two ribbons. The two HXR kernels also exhibit eastward elongation motion during the first 2-3 minutes at an average speed of 55 and $45 \mathrm{~km} \mathrm{~s}^{-1}$ in the positive and negative fields, respectively (Cheng et al. 2012). Ten minutes later, a second episode of energy release takes place in the east of the active region, and both ribbons appear to spread mostly eastward, at speeds of 8 and $12 \mathrm{~km} \mathrm{~s}^{-1}$, in the positive and negative fields, respectively. For this active region, the macroscopic electric current runs eastward. Therefore, the overall elongation of the ribbons, at the initial stage of energy release, is parallel to the current direction. Cheng et al. (2012) also estimated the inclination in these two stages, using both UV and HXR data; this angle is $40^{\circ}$ and $75^{\circ}$, respectively, at the start of each of the two stages of energy release.

\subsection{Properties of Ribbon Elongation and Magnetic Fields}

These observations, albeit from a rather small sample, demonstrate a variety of ribbon elongation patterns. Ribbons may elongate in a single direction, either parallel or antiparallel to the current, or bidirectionally. The parallel spreading occurs at a range of speeds from a few kilometers per second to more than one hundred kilometers per second. These properties might be related to properties of the magnetic field or electric current in the corona. The inclination angle of the postreconnection connectivity is a frequently measured property. Apart from this, we also estimate other properties, including the mean photospheric longitudinal magnetic field strength $\left\langle B_{\mathrm{ph}}\right\rangle$ at the location of the initial brightening where elongation starts, the mean gradient of the photospheric magnetic field strength $\left\langle\nabla_{\|}\left|B_{\mathrm{ph}}\right|\right\rangle$ along the ribbon direction, and the mean total magnetic field strength in the corona $\left\langle B_{c}\right\rangle$ at the PIL and its gradient along the PIL. The coronal magnetic field is estimated by a potential field extrapolation at the approximate height of the flare loop top, estimated from the footpoint separation. It is understood that all of these measurements, especially the coronal magnetic field, carry large uncertainties. The uncertainties in $\left\langle B_{\mathrm{ph}}\right\rangle$ and $\left\langle B_{c}\right\rangle$ are quoted as the standard deviation of the measured field in multiple pixels along the newly brightened ribbon or the coronal PIL, and the uncertainty in $\left\langle\nabla_{\|}\left|B_{\mathrm{ph}}\right|\right\rangle$ is simply the standard deviation of the linear fit to derive the gradient, which is the lower limit of real uncertainties.

These measurements for the six events, including different episodes of energy release for some events, are listed in Table 1, and a comparison of their properties is given in Figure 10, showing the unsigned or signed ribbon elongation speed versus various magnetic properties estimated above.

From this small sample categorized into three groups (parallel, anti-parallel, or bidirectional elongation), it first appears that the two events ( 3 and 5) exhibiting bidirectional elongation have the strongest shear. This would indicate a large relative guide field in a $2.5 \mathrm{D}$ approximation with $B_{g} / B_{o}$ greater than 3 in the first few minutes. For the other four events, with two exhibiting parallel elongation and the other two antiparallel elongation, there is no clear distinction in the shear: all of them have a weak to moderate shear, with $B_{g} / B_{o}$ ranging between 0.3 and 1.2. In terms of elongation speed, there is a vague trend that events (or locations) with greater shear move faster. The strong shear events (3 and 5), though exhibiting a variety of elongation speeds at different locations, have maximal speeds close to $100 \mathrm{~km} \mathrm{~s}^{-1}$, whereas the slowest motion is measured in the second stage of event 6 $\left(8-12 \mathrm{~km} \mathrm{~s}^{-1}\right)$, which is associated with the weakest shear 

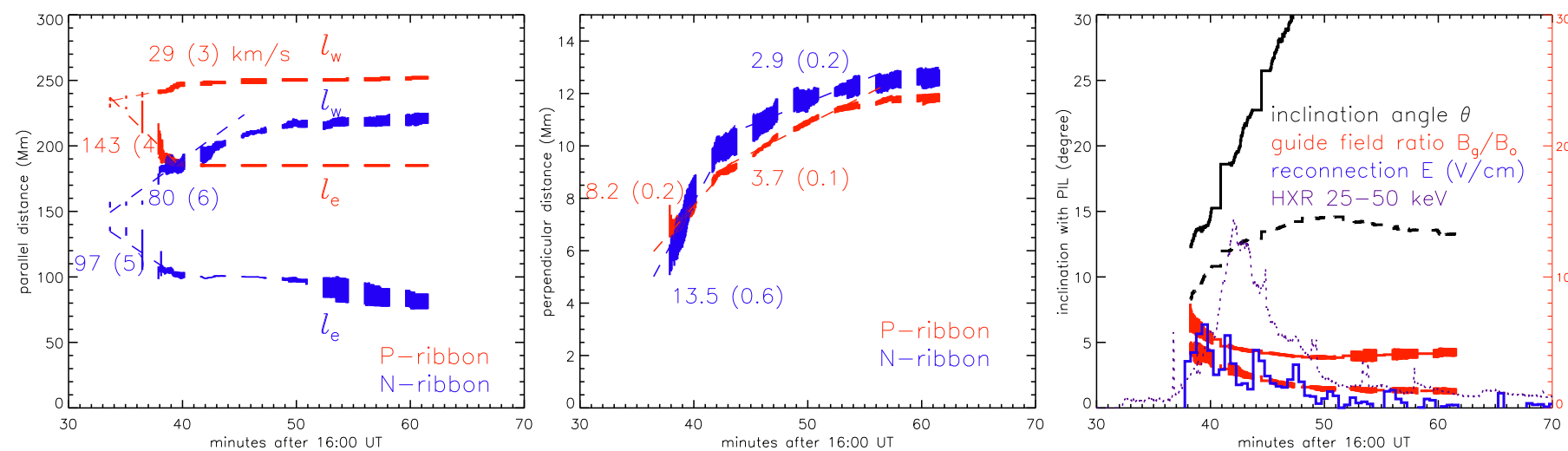

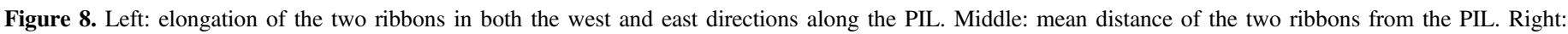
inclination angle of the flare (see text), the presumed $B_{g} / B_{o}$, the mean electric field given by $\langle E\rangle=v_{\perp} B_{n}$, and the HXR $25-50 \mathrm{keV}$ light curve.

$\left(\theta_{f}=75^{\circ}\right.$, and $\left.B_{g} / B_{o}=0.3\right)$. However, for the entire sample, the correlation between the speed and shear is low (Figure 10(a)).

Another possible factor in governing the elongation speed is the coronal magnetic field strength itself. This comparison is given in Figure 10(b), showing that the ribbon speed is roughly anti-correlated with the total magnetic field strength at the approximate height of the loop top. This trend is partly governed by the two extreme cases, the second stage of event 6 with the slowest motion in the strongest magnetic fields, and event 2 with the lowest mean magnetic field but a rather high speed close to $100 \mathrm{~km} \mathrm{~s}^{-1}$. This is so far the strongest trend in all these comparisons. It is not clear why the elongation speed is nearly anti-correlated with the coronal magnetic field.

If we consider the guide-field strength to be the product of the coronal magnetic field strength and the cosine of the inclination angle, the correlation in Figure 10(a) and the anticorrelation in Figure 10(b) lead to a scatter between the measured speed and estimated guide-field strength. Therefore, the present observations, which carry a large uncertainty in our estimate of $B_{g}$, do not support the scenario that elongation is solely governed by Alfvén waves. Furthermore, the maximum measured speed is nearly an order of magnitude smaller than the characteristic coronal Alfvén speed at the coronal magnetic field strength presented in Table 1.

Since the coronal magnetic field in the present study is estimated very roughly from the potential field extrapolation, we also examine the mean photospheric magnetic field at the locations of the initial brightenings. Note that only the longitudinal magnetic field strength is measured, because four events are observed by MDI, which does not have vector field measurements. Figure 10(c) shows that the speed appears to be anti-correlated with $\left\langle\left|B_{\mathrm{ph}}\right|\right\rangle$, though to less of an extent than in Figure 10(b).

It is noted that the motion of the conjugate ribbon fronts in opposite magnetic fields is mostly asymmetric. Since the same amounts of positive and negative magnetic flux participate in reconnection, ribbons should move faster in weaker magnetic fields; such a flux balance rule, namely $v_{+} B_{+} \approx v_{-} B_{-}(+$and indicate properties in the positive and negative magnetic fields, respectively), is observed within a factor of two for these six events, if we ignore the perpendicular motion. This fact partly contributes to the anti-correlation between $v_{\|}$and $\left\langle\left|B_{\mathrm{ph}}\right|\right\rangle$ shown in Figure 10(c), but cannot explain the stronger anti-correlation of the elongation speed with the coronal magnetic field. It is also noted that bidirectional elongation (in events 3 and 5) from one location is usually not symmetric. To understand whether these asymmetries are due to the local magnetic field distribution, Figure 10(d) compares the signed speed with the gradient of the local magnetic field strength along the PIL. A plausible hypothesis could be that ribbon spreading tends to proceed in the direction the magnetic field decreases. Therefore, in Figure 10(d), the signed speed is plotted with respect to the signed magnetic gradient, with the positive sign indicating ribbon elongation or magnetic field decrease in the direction of (i.e., parallel to) the electric current, and negative sign indicating ribbon elongation or magnetic field strength decrease in the direction opposite to (or anti-parallel to) the electric current. Figure 10(d), although exhibiting a weak correlation between the two, shows very large scatter and low significance. In this sample, the strongest supporting case is the positive ribbon in event 3 , which spreads at a high speed away from the sunspot with quickly decreasing magnetic field; however, in this same event, negative ribbons appear to spread along the direction where the magnetic field increases. The second stage of event 6 shows a similar counterexample, with the ribbon spreading in the direction along which the magnetic field increases quickly. Event 2 shows an extreme case of fast spreading of ribbons in a pair of plages with a mean magnetic gradient close to zero. The rather random distribution of the elongation speed with respect to $\left\langle\nabla_{\|}|| B_{\mathrm{ph}} \mid\right\rangle$ suggests that the local magnetic gradient is not a governing factor for ribbon elongation speed.

The mean gradient of the coronal magnetic field along the PIL is also estimated. The coronal field from the extrapolation exhibits a much smoother distribution along the PIL but a similar trend of gradients to that in the photosphere, although the magnitude of the gradient is smaller by one to two orders of magnitude (not shown).

Furthermore, the gradient of the photospheric magnetic field strength in the direction perpendicular to the PIL is estimated at the locations of initial brightenings (not shown). It is observed that ribbon brightening tends to start from the edge of a plage or penumbra close to the PIL; therefore, in these regions of initial brightening, the unsigned magnetic field strength increases away from the PIL. The magnitude of this perpendicular gradient ranges from a few tens to a few hundreds Gauss per Mm, about an order of magnitude greater than the parallel gradient in the photosphere. However, it is less clear how to relate the locally measured perpendicular gradient of the photospheric magnetic field with the coronal configuration around the reconnection region, even in a $2.5 \mathrm{D}$ 

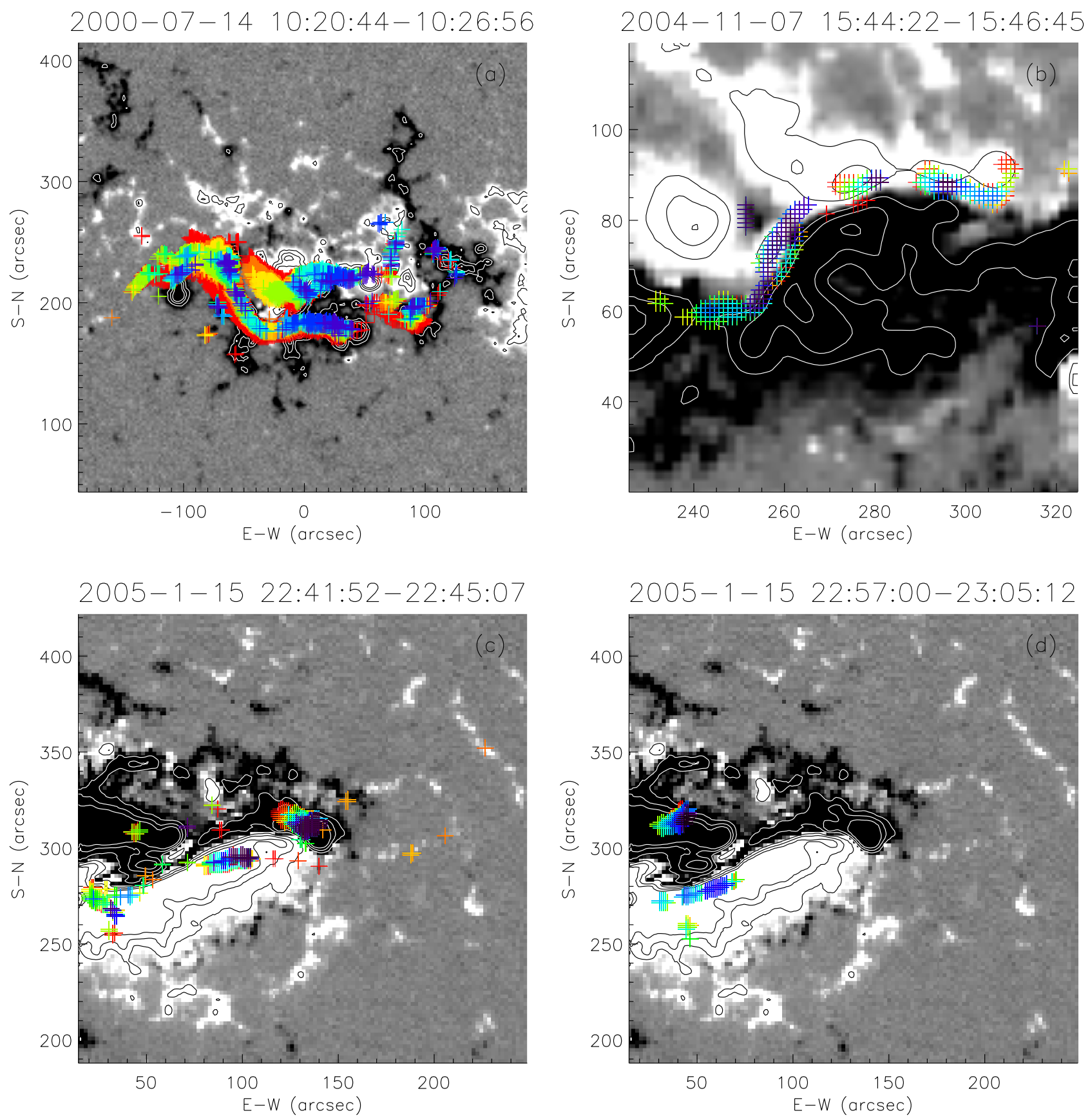

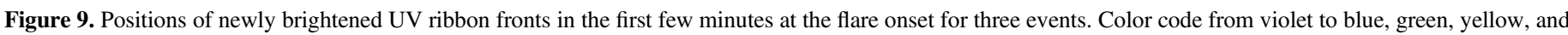
orange indicates the time of the ribbon fronts.

approximation. These measurements also do not illustrate any pattern of correlation or anti-correlation with ribbon elongation in this sample.

Liu et al. (2010) also measured the $\mathrm{H} \alpha$ and hard X-ray footpoint motion in event 6 and found that $\mathrm{H} \alpha$ and hard X-ray kernels spread in the direction along which the overlying magnetic field decays with height more quickly. Our analysis with this small sample, however, does not reveal a correlation between the direction of ribbon spreading and the decay index of the overlying field along the PIL.

In addition, Table 1 records the mean speed of the perpendicular expansion $v_{\perp}$ of the entire ribbon when it is fully formed. This average speed ranges from a few to up to $10 \mathrm{~km} \mathrm{~s}^{-1}$, generally smaller than $v_{\|}$, and we do not find a correlation between the elongation speed $v_{\|}$and the perpendicular speed $v_{\perp}$.

In summary, for the given small sample of six two-ribbon flares, the ribbon elongation speed appears to be anti-correlated with the photospheric magnetic field strength as well as the mean coronal magnetic field along the PIL. On the other hand, we do not find that magnetic properties or motion speeds associated with parallel elongation are substantially different from those of anti-parallel elongation.

\section{Summary and Discussion}

We present an analysis of apparent motion of flare ribbon brightenings along the magnetic PIL, the so-called "elongation" motion, in the early phase of three flares observed in the UV 

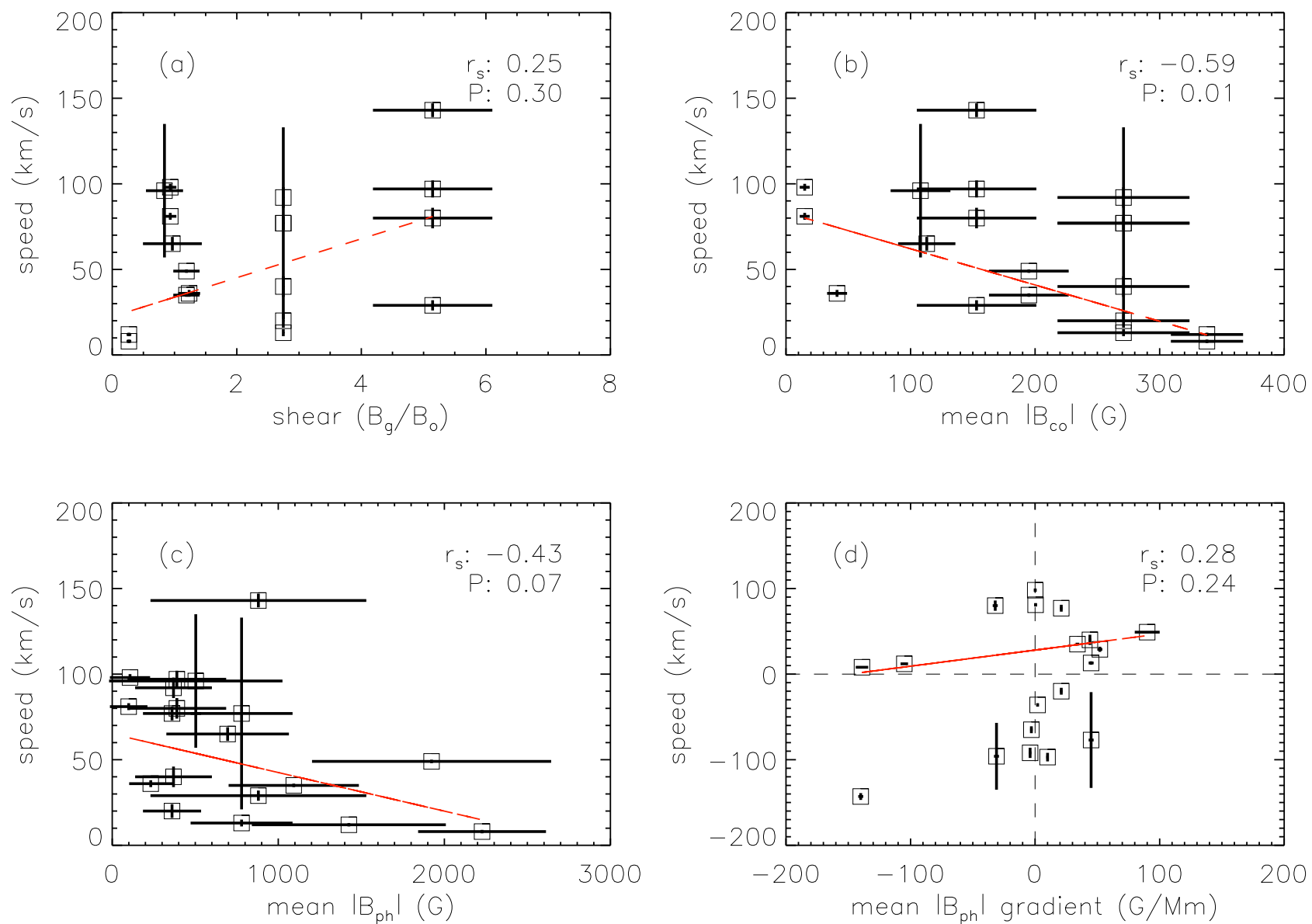

Figure 10. Scatter plot of the ribbon elongation speed vs. magnetic properties: (a) unsigned speed vs. the magnetic shear in terms of the ratio $B_{g} / B_{o}$; (b) unsigned speed vs. the mean coronal magnetic field strength; (c) unsigned speed vs. the mean photospheric magnetic field strength; (d) signed speed with respect to the local magnetic gradient along the PIL (see text). In each panel, the red dashed line shows the linear fit to the data, and $r_{s}$ and $P$ give the Spearsman rank correlation coefficient and the significance of its deviation from zero, respectively.

$1600 \AA$ bandpass. They each exhibit a different pattern of ribbon elongation. In one event, ribbon brightening spreads along the PIL in the direction opposite to the macroscopic electric current in the corona (anti-parallel elongation) for as long as one hour at a mean speed of $11 \mathrm{~km} \mathrm{~s}^{-1}$. Another event exhibits two ribbons spreading quickly in the same direction as the current (parallel elongation) at a mean speed of nearly $100 \mathrm{~km} \mathrm{~s}^{-1}$ for about 10 minutes. In the third event, brightening spreads quickly and bidirectionally. The first two flares were also observed in the EUV bandpasses by AIA, showing ordered spreading of flare EUV loops along the PIL after the ribbon brightening, at mean speeds systematically smaller than the ribbon spreading. These observations confirm the "zipper" pattern of elongation motion in both flare ribbons and flare loops produced by successive reconnection energy releases along the PIL.

We measure the inclination angle $\theta$ of the line connecting conjugate footpoints with respect to the PIL of the longitudinal magnetic field of the photosphere. With flare loops observed in the EUV $171 \AA$ passband by AIA, this angle is also measured as the inclination of the observed loop tops with respect to the PIL. The two measurements are consistent with each other, both showing gradually decreased shear as the flare evolves and both ribbons and loops spread along the PIL. The measured flare shear is also larger (i.e., smaller $\theta$ ) than that of the potential field, suggesting that flare loops usually are not yet relaxed to a potential configuration. With a $2.5 \mathrm{D}$ approximation of the flare arcade configuration, we estimate the magnetic guide field $B_{g}$, the component along the current (and PIL) that does not participate in reconnection, relative to the reconnection outflow field $B_{o}$ using the relation $B_{g} / B_{o} \approx \cot \theta$. We find that the event with bidirectional elongation has a strong shear with $B_{g} / B_{o} \approx 5$, and the other events with unidirectional elongation have a moderate shear with $B_{g} / B_{o} \approx 0.4-1.2$.

We review properties of elongation in another three $\mathrm{X}$-class flares analyzed in previous studies, and compare these properties with magnetic properties in flare regions for all six events. It is observed that, in this small sample, ribbon elongation speed is greater in events with a weaker mean coronal or photospheric magnetic field, but is less correlated with the inclination angle $\theta$ or the magnetic gradient $\left\langle\nabla_{\|}|B|\right\rangle$ along the PIL measured either locally in the photosphere or in the corona.

These results demonstrate the difficulty of identifying physical mechanisms governing the ribbon elongation motion. As much as we have attempted to infer reconnection properties from signatures in the lower atmosphere, where the magnetic field can be measured, there are large uncertainties in deriving the magnetic field in the corona. The difficulties are even greater in inferring properties within the reconnecting current sheet. The two events of bidirectional elongation exhibit a strong shear with $B_{g} / B_{o}>3$, which might support the scenario of Alfvén waves. On the other hand, in these events, the motion 
speed of $\leqslant 100 \mathrm{~km} \mathrm{~s}^{-1}$ is an order of magnitude smaller than the coronal Alfvén speed for the given mean coronal magnetic field in Table 1 (if $n \sim 10^{9} \mathrm{~cm}^{-3}$ ). It is also shown that, for the entire sample, the present measurements (with very large uncertainties in the coronal magnetic field) cannot establish the evidence that the elongation speed $v_{\|}$scales with the guide-field strength $B_{g}$. Alternatively, because of the large uncertainty in the coronal magnetic field measurements, we may estimate the guide-field strength based on the ribbon elongation speed. This yields $B_{g} \approx 2 \mathrm{G}$ for the measured fastest speed at about $100 \mathrm{~km} \mathrm{~s}^{-1}$. At the strongest shear $\theta \approx 10^{\circ}-20^{\circ}$, we find the outflow magnetic field component to be less than $1 \mathrm{G}$, which is significantly smaller than the coronal field from extrapolation.

The other population of events of parallel or anti-parallel elongation both exhibit a range of initial motion speed from a few to nearly a hundred kilometers per second with $B_{g} / B_{o} \approx 0.3-1.2$. If we consider these motions to be produced by drifting of current-carrying particles, the inferred thickness of the reconnection current sheet is of order $10^{5}-10^{6} \mathrm{~cm}$ (again if $n \sim 10^{9} \mathrm{~cm}^{-3}$ ). It is interesting to see that whereas this scale is larger, by $2-3$ orders of magnitude, than some theoretical estimates (such as in the Sweet-Parker and Hall reconnection models), it is smaller by 1-2 orders of magnitude than the reported thickness of long current sheets trailing behind CMEs (Lin et al. 2015 and references therein). The thickness of a flare current sheet remains a matter of debate (e.g., Lin et al. 2015).

Results from this small sample also show an anti-correlation between elongation speed and the magnetic field strength. The anti-correlation shown for the photospheric magnetic field is partly attributed to the reconnection flux balance between positive and negative fields. Yet the anti-correlation with the coronal magnetic field strength is not understood. Energy release in strong magnetic fields tends to be greater, which might in general require a fast reconnection or a large characteristic speed in the system. The mean magnetic field strength in this sample varies from one event to another by one and a half orders of magnitude; the total reconnection flux as well as the peak reconnection rate (in units of Maxwell per second) in these events also vary by one and a half orders of magnitude, with flares in stronger magnetic fields indeed exhibiting greater reconnected flux and reconnection rate. On the other hand, measurements in this paper show that flares in stronger magnetic fields do not spread faster.

All of the events in this sample are accompanied by coronal mass ejections, but most of them are not accompanied by erupting filaments. The SOL2000-07-14 flare starts at the location where a filament lifts off, and for the SOL2005-01-15 event, Liu et al. (2010) suggested that flare kernels spread in the direction along which the overlying magnetic field decreases with height more rapidly. But for most of these events, we do not find a clear association between the location or elongation speed of the initial ribbon brightening and filament or CME dynamics. On the other hand, Hu et al. (2014) and Priest \& Longcope (2017) have discussed how the geometry and sequence of reconnection as reflected in ribbon motion may lead to the formation of flux ropes.

To achieve an understanding of possible governing mechanisms, it will be helpful to apply the analysis to a larger sample of ribbon elongation in both eruptive flares and compact flares (e.g., Veronig \& Polanec 2015) with simple configurations. Since a more accurate inference of coronal magnetic fields cannot be achieved at present, we hope that analysis of a larger sample will help clarify whether there are trends relating ribbon motion direction and speed with the shear that will allow us to test the proposed mechanisms.

We also acknowledge that the magnetic configuration at or near the reconnection site is quite different from a potential field. For instance, reconnection may occur at the top of a cusp structure, at an altitude possibly much greater than the top of flare loops, which have retracted from the reconnection site. In such a circumstance, the field strength may be much smaller than estimated in this paper, and the characteristic speed of reconnection spread could be smaller as well. To verify this scenario, it is useful to explore suitable and simultaneous coronal observations from a different vantage point, such as those by STEREO, which may allow us to observe the cusp structure and estimate the height and magnetic field there.

The authors thank the referee for a careful review and constructive comments that helped improve the paper. J.Q., D. W.L., and P.A.C. gratefully acknowledge support by NSF SHINE collaborative grant AGS-1460059. SDO is a mission of NASA's Living With a Star Program.

\section{References}

Aschwanden, M. J., \& Alexander, D. 2001, SoPh, 204, 91

Aulanier, G., Janvier, M., \& Schmieder, B. 2012, A\&A, 543, A110

Aulanier, G., Pariat, E., Démoulin, P., \& DeVore, C. R. 2006, SoPh, 238, 347 Bogachev, S. A., Somov, B. V., Kosugi, T., \& Sakao, T. 2005, ApJ, 630, 561 Cheng, J. X., Kerr, G., \& Qiu, J. 2012, ApJ, 744, 48

Cheng, J. X., \& Qiu, J. 2016, ApJ, 825, 37

Coyner, A. J., \& Alexander, D. 2009, ApJ, 705, 554

Dudík, J., Janvier, M., Aulanier, G., et al. 2014, ApJ, 784, 144

Fletcher, L., \& Hudson, H. 2001, SoPh, 204, 69

Fletcher, L., Pollock, J. A., \& Potts, H. E. 2004, SoPh, 222, 279

Forbes, T. G., \& Lin, J. 2000, JASTP, 62, 1499

Grigis, P. C., \& Benz, A. O. 2005, ApJL, 625, L143

Handy, B. N., Acton, L. W., Kankelborg, C. C., et al. 1999, SoPh, 187, 229

Hu, Q., Qiu, J., Dasgupta, B., Khare, A., \& Webb, G. M. 2014, ApJ, 793, 53 Huba, J. D., \& Rudakov, L. I. 2002, PhPl, 9, 4435

Huba, J. D., \& Rudakov, L. I. 2003, PhPl, 10, 3139

Inglis, A. R., \& Gilbert, H. R. 2013, ApJ, 777, 30

Isobe, H., Shibata, K., \& Machida, S. 2002, GeoRL, 29, 2014

Isobe, H., Takasaki, H., \& Shibata, K. 2005, ApJ, 632, 1184

Janvier, M., Aulanier, G., Pariat, E., \& Démoulin, P. 2013, A\&A, 555, A77

Karimabadi, H., Krauss-Varban, D., Huba, J. D., \& Vu, H. X. 2004, JGR, 109, A09205

Katz, N., Egedal, J., Fox, W., et al. 2010, PhRvL, 104, 255004

Kawaguchi, I., Kurokawa, H., Funakoshi, Y., \& Nakai, Y. 1982, SoPh, 78, 101

Kazachenko, M. D., Canfield, R. C., Longcope, D. W., et al. 2009, ApJ, 704, 1146

Kitahara, T., \& Kurokawa, H. 1990, SoPh, 125, 321

Kosugi, T., Makishima, K., Murakami, T., et al. 1991, SoPh, 136, 17

Krucker, S., Hurford, G. J., \& Lin, R. P. 2003, ApJL, 595, L103

Lapenta, G., Krauss-Varban, D., Karimabadi, H., et al. 2006, GeoRL, 33, 10102

Lee, J., \& Gary, D. E. 2008, ApJL, 685, L87

Lemen, J. R., Title, A. M., Akin, D. J., et al. 2012, SoPh, 275, 17

Li, L., \& Zhang, J. 2009, ApJ, 690, 347

Lin, J., Murphy, N. A., Shen, C., et al. 2015, SSRv, 194, 237

Lin, R. P., Dennis, B. R., Hurford, G. J., et al. 2002, SoPh, 210, 3

Liu, C., Lee, J., Jing, J., et al. 2010, ApJL, 721, L193

Liu, C., Lee, J., Yurchyshyn, V., et al. 2007, ApJ, 669, 1372

Liu, W.-J., Qiu, J., Longcope, D. W., \& Caspi, A. 2013, ApJ, 770, 111

Longcope, D., Beveridge, C., Qiu, J., et al. 2007, SoPh, 244, 45

Masson, S., Pariat, E., Aulanier, G., \& Schrijver, C. J. 2009, ApJ, 700, 559

Poletto, G., \& Kopp, R. A. 1986, in The Lower Atmosphere of Solar Ares;

Proc. Solar Maximum Mission Symp., ed. D. F. Neidig (Sunspot, NM:

National Solar Observatory), 453 DOE-sponsored research

Priest, E., \& Longcope, D. 2017, arXiv:1701.00147

Priest, E. R., \& Démoulin, P. 1995, JGR, 100, 23443 
Qiu, J. 2009, ApJ, 692, 1110

Qiu, J., Lee, J., Gary, D. E., \& Wang, H. 2002, ApJ, 565, 1335

Qiu, J., Liu, W., Hill, N., \& Kazachenko, M. 2010, ApJ, 725, 319

Qiu, J., \& Longcope, D. W. 2016, ApJ, 820, 14

Qiu, J., \& Yurchyshyn, V. B. 2005, ApJL, 634, L121

Savcheva, A., Pariat, E., McKillop, S., et al. 2015, ApJ, 810, 96

Shay, M. A., Drake, J. F., Swisdak, M., Dorland, W., \& Rogers, B. N. 2003, GeoRL, 30, 1345

Shepherd, L. S., \& Cassak, P. A. 2012, JGRA, 117, 10101

Su, Y., Golub, L., \& Van Ballegooijen, A. A. 2007, ApJ, 655, 606
Temmer, M., Veronig, A. M., Vršnak, B., \& Miklenic, C. 2007, ApJ, 654, 665

Tripathi, D., Gibson, S. E., Qiu, J., et al. 2009, A\&A, 498, 295

Tripathi, D., Isobe, H., \& Mason, H. E. 2006, A\&A, 453, 1111

Tsuneta, S., Acton, L., Bruner, M., et al. 1991, SoPh, 136, 37

Veronig, A. M., \& Polanec, W. 2015, SoPh, 290, 2923

Vorpahl, J. A. 1976, ApJ, 205, 868

Yang, Y.-H., Cheng, C. Z., Krucker, S., Lin, R. P., \& Ip, W. H. 2009, ApJ, 693, 132

Yurchyshyn, V., Liu, C., Abramenko, V., \& Krall, J. 2006, SoPh, 239, 317 